IZA DP No. 104

Different Skill Levels and Firing Costs in a Matching Model with Uncertainty An Extension of Mortensen and Pissarides (1994)

Stephan Kohns

J anuary 2000 


\title{
Different Skill Levels and Firing Costs in a Matching Model with Uncertainty - An Extension of Mortensen and Pissarides (1994)
}

\author{
Stephan Kohns \\ University of Bonn and IZA, Bonn \\ Discussion Paper No. 104 \\ January 2000 \\ IZA \\ P.O. Box 7240 \\ D-53072 Bonn \\ Germany \\ Tel.: +49-228-3894-0 \\ Fax: +49-228-3894-210 \\ Email: iza@iza.org
}

This Discussion Paper is issued within the framework of IZA's research area Mobility and Flexibility of Labor Markets. Any opinions expressed here are those of the author(s) and not those of the institute. Research disseminated by IZA may include views on policy, but the institute itself takes no institutional policy positions.

The Institute for the Study of Labor (IZA) in Bonn is a local and virtual international research center and a place of communication between science, politics and business. IZA is an independent, nonprofit limited liability company (Gesellschaft mit beschränkter Haftung) supported by the Deutsche Post AG. The center is associated with the University of Bonn and offers a stimulating research environment through its research networks, research support, and visitors and doctoral programs. IZA engages in (i) original and internationally competitive research in all fields of labor economics, (ii) development of policy concepts, and (iii) dissemination of research results and concepts to the interested public. The current research program deals with (1) mobility and flexibility of labor markets, (2) internationalization of labor markets and European integration, (3) the welfare state and labor markets, (4) labor markets in transition, (5) the future of work, (6) project evaluation and (7) general labor economics.

IZA Discussion Papers often represent preliminary work and are circulated to encourage discussion. Citation of such a paper should account for its provisional character. 


\title{
ABSTRACT \\ Different Skill Levels and Firing Costs in a Matching Model with Uncertainty - An Extension of Mortensen and Pissarides (1994)
}

A matching model in the line of Mortensen and Pissarides (1994) is augmented with a lowskill labor market and firing costs. It is shown that even with flexible wages unemployment is higher among the low-skilled and increases with skill-biased technological change. The two main reasons are that their jobs have a shorter life expectancy than in the labor market for the skilled, increasing the inflow into unemployment, and that the jobs are less profitable, resulting in a smaller outflow from unemployment. Firing costs increase employment security among existing jobs, but the unskilled are likely to profit less than the skilled, and the availability of new jobs decreases in both sectors. Within the present framework the effect of firing costs on unemployment is ambiguous, but unemployment spells are shown to be longer with higher firing costs. The implications of explicitly introducing business cycles into the model are considered, too.

JEL Classification: J23, J32, J63, J64

Keywords: Firing costs, low-skill unemployment, dual labor market, vacancies

\author{
Stephan Kohns \\ Institute of International Economics \\ University of Bonn \\ Lennéstr. 37 \\ 53113 Bonn \\ Germany \\ Tel: +49-228-73-9234 \\ Email:kohns@iiw.uni-bonn.de
}




\section{Introduction}

For a given wage, labor demand and thereby also the dynamics of employment and unemployment are to a large extent determined by three factors: adjustment costs (mainly for hiring and firing), uncertainty about future profits due to shocks to demand or productivity, and the skill level of the employed.

There is already a large literature that investigates the influence of one or the other of these factors. Bentolila and Bertola (1990) use methods from control theory to analyze the effects of linear adjustment costs in a model with homogeneous labor and demand shocks, and Ross (1998) extends their analysis by introducing a second factor that can be adjusted costlessly. Both models are partial-equilibrium models. It is not possible to derive a closed form solution for labor demand, but at least the solution can be characterized qualitatively: Contrary to convex costs, linear costs lead to lumpy adjustment of labor at the level of the firm, which also seems to be more in line with empirical evidence (see e.g. Huizinga and Schiantarelli (1992) and the overview given in Hamermesh and Pfann (1996)).

Bentolila and Sain-Paul (1992) and Saint-Paul (1996b, chp. 3, 4) analyze the interdependence of the demand for workers with permanent and fixed-term contracts in a partial-equilibrium model with productivity shocks and adjustment costs only for the permanently employed. In this framework, the workers on fixed-term contracts bear the burden of adjustment in the case of negative productivity shocks. Saint-Paul (1996b, chp. 7) derives similar results under slightly more restrictive assumptions but with endogenous wages and labor supply. In principle, it is possible to assign different skill levels to the two types of workers, but using this as a framework to analyze the differences between skilled and low-skilled labor leads to untenable results, since both types of labor are, apart from the productivity differential, perfect substitutes. Saint-Paul (1996a), (1996b, Kapitel 9) explicitly differentiates between skilled and unskilled labor in a deterministic matching model, focusing in particular on the displacement of

low-skilled by skilled workers, the role of skill-biased technological change and the effects of firing costs for the low-skilled.

This paper tries to incorporate all the three factors mentioned above in a single 
model, using the seminal paper by Mortensen and Pissarides (1994) with homogeneous workers as a starting point. In their model, productivity is job specific and subject to idiosyncratic as well as global changes, i.e. the productivity of an individual or of all jobs changes stochastically. As soon as the productivity of a given job falls below a certain, endogenously determined threshold, the job is dissolved without costs. The present model extends this framework by introducing two skill-levels as well as relaxing the assumption of no firing costs.

Section 2 presents the basic setup of the model and the labor market for the two skill-levels. Section 3 introduces firing costs in both markets. The implications of explicitly modelling business cycles are investigated in section 4 . Section 5 concludes. Calculations that require more room or that are particularly tedious are relegated to the appendix.

\section{Basic structure and steady-state analysis}

\subsection{Basic structure of the model}

Consider an economy in continuous time. Firms can employ two types of workers that differ with respect to their skill level (e.g. white- and blue-collar workers), and it is assumed that a higher skill level translates into a higher level of productivity. Variables that are particular to one of the groups will be denoted with superscripts $s$ and $l$ for skilled and low-skilled workers, respectively.

Vacancies posted by firms in order to employ workers have to be skill-specific, i.e. it is not possible to put a blue-collar worker on a position designed for white-collar workers and vice versa. ${ }^{1}$ Unfilled vacancies cost $c$ per unit of time (all prices and assets are measured in units of output). Only the unemployed search for jobs, i.e. we neglect on the job search. Let $v^{\nu}$ and $u^{\nu}, \nu=s, l$, denote the number of vacancies and unemployed, respectively, on market segment $\nu$. The ratio $v^{\nu} / u^{\nu}$ will be denoted by the variable $\theta^{\nu}$ and measures the tightness of labor market segment $\nu$, as perceived by a firm.

In both labor market segments, the unemployed and vacancies are matched ac-

\footnotetext{
${ }^{1}$ As long as workers cannot apply for several vacancies simultaneously, the only assumption that is really needed is that low-skilled workers are unable to occupy a job designed for the skilled, see page 14 .
} 
cording to the same matching function $m\left(v^{\nu}, u^{\nu}\right)$ that is homogeneous of degree one. Dividing $m(\cdot, \cdot)$ by $v^{\nu}$ yields the rate $\phi\left(\theta^{\nu}\right):=\frac{m\left(v^{\nu}, u^{\nu}\right)}{v^{\nu}}=m\left(1, \frac{u^{\nu}}{v^{\nu}}\right)=$ $m\left(1,\left(\theta^{\nu}\right)^{-1}\right)$ at which vacancies are filled. It follows that $\phi^{\prime}\left(\theta^{\nu}\right)<0$ and, denoting the elasticity of $\phi\left(\theta^{\nu}\right)$ with $-\eta$, that $0<\eta<1$. These two properties will prove to be important for the following analysis. The first simply means that with increasing tightness of the labor market, it becomes more and more difficult to fill a given vacancy (even though the absolute number of filled vacancies may be higher). The inelastic reaction of $\phi(\cdot)$ is simply a result of the constant returns assumption. Note that $\eta$ is generally not constant for different values of $\theta^{\nu}{ }^{2}$

For the skilled, the productivity of a given job can be decomposed additively into a global and an idiosyncratic, i.e. job-specific, component. ${ }^{3}$ Global productivity is denoted by $p$, whereas the job-specific component is the product of a constant parameter $\sigma$ and an idiosyncratic, zero-mean shock $\varepsilon$. The standard deviation of $\varepsilon$ is normalized to one, so that $\sigma$ is the standard deviation of the job-specific component. The distribution of $\varepsilon$ can be described by a differentiable distribution function $F(\varepsilon)$. The maximum value of the idiosyncratic shock is $\varepsilon_{u}$, so that $F\left(\varepsilon_{u}\right)=1$, and this is also the value $\varepsilon$ takes for every newly created job (i.e. for a vacancy that has just been filled). A possible interpretation would be that new jobs are always equipped with the most productive technology. All the jobs are subject to random changes in the job-specific component of productivity. The occurrence of these productivity changes is described by a Poisson process with parameter $\lambda$. When an adjustment takes place, a new value of $\varepsilon$ is drawn according to the distribution function $F(\varepsilon) .{ }^{4}$ When the idiosyncratic component drops below a threshold $\varepsilon_{d}^{s}$, the job is disbanded at not cost and the worker gets unemployed. The superscript $s$ indicates that the threshold may differ in the two

\footnotetext{
${ }^{2} \mathrm{~A}$ constant elasticity follows for example from a Cobb-Douglas specification of the matching function $m(\cdot, \cdot)$. This is not an unfamiliar choice, see e.g. the matching model by Pissarides (1992) on the role of long-term unemployment and the loss of human capital. The CobbDouglas specification is also supported by the empirical analysis of Burda and Wyplosz (1994) (against the alternative of a CES-specification), but the authors find only mixed evidence for the hypothesis of constant returns (rejection for France and Spain, acceptance for Germany and the UK).

${ }^{3}$ The two expressions "idiosyncratic" and "job-specific" will be used interchangeably.

${ }^{4}$ Within the present continuous-time setting, two particularly useful properties of Poisson processes are that firstly, the probability of one happening of a certain event in a small time interval $d t$ equals $\lambda d t$ and secondly, the occurrence of events in non-overlapping time intervals is independent, meaning that we don't have to care about when or how often the idiosyncratic component changed its value before.
} 
labor market segments.

The above remarks imply that the overall productivity of a skilled worker equals $p+\sigma \varepsilon$. Low-skilled workers are assumed to be less productive on average. This is modelled as a productivity differential $\rho$ in the idiosyncratic component, leading to a total productivity of $p+\sigma(\varepsilon-\rho)$. The probability structure of the idiosyncratic shocks and of the Poisson process governing their occurrence is assumed to be equal in both labor market segments. ${ }^{5}$

Additional costs like interest payments on rented capital are not explicitly modelled, but as long as they are fixed for every job, they can be captured by the parameter $p$.

Workers receive wages $w^{\nu}(\varepsilon)$ which are flexible in the sense that their payment depends on the current productivity of the job. If they are unemployed, they get a given benefit $b .^{6}$

Apart from common parameters like the global productivity component $p$ or the $\operatorname{costs} c$ of posting a vacancy, the labor market segments are implicitly linked by the no-arbitrage-condition that the expected values of vacancies for skilled and low-skilled workers have to be equal, i.e. a firm is indifferent between creating another job for a skilled or for a low-skilled worker. Otherwise, it would be profitable to shift vacancies from one segment to the other.

The following subsection introduces the labor market segment of the skilled and analyzes the dependence of the equilibrium outcomes on changes in the exogenous parameters, following mainly the analysis of Mortensen and Pissarides (1994). The low-skill tier of the labor market and its differences from the first tier are

\footnotetext{
${ }^{5}$ It would also be possible to model the lower productivity of the low-skilled as a discount on the global productivity component $p$, so that their total productivity would equal $p+\sigma \varepsilon-\rho$. This would facilitate the analysis - the low-skill segment could be treated as a 'normal' segment with a lower global productivity -, but it would also blur the distinction between the job-specific and the global component.

Using the same distribution (instead of a distribution shifted by $\rho$ to the left) facilitates a comparison of the results in the two labor market segments.

Note also that the extension to more than two skill levels by introducing productivity differentials $\rho_{1}, \rho_{2}, \ldots, \rho_{k}$ is straightforward.

${ }^{6}$ Skill-dependent unemployment benefits could be introduced as well but have been neglected for simplicity. If they are modelled, one has to take care to ensure that the condition $b^{s}<b^{l}+\sigma \rho$ holds. Otherwise, low-skilled workers would be more valuable to the firm than the skilled, as can be seen from the following equations.
} 
discussed in subsection 2.3.

\subsection{The labor market for the skilled}

Let $V^{s}$ denote the value of a vacancy and $J^{s}(\varepsilon)$ the value of a filled job with job-specific productivity $\varepsilon$, in both cases as perceived by the firm. Assuming a constant interest rate $r$, we have

$$
r V^{s}=\phi\left(\theta^{s}\right)\left[J^{s}\left(\varepsilon_{u}\right)-V^{s}\right]-c,
$$

which states that the return of a vacancy per unit of time equals the expected gain, if the vacancy is filled, minus the cost of keeping the vacancy open. The expected gain depends on the rate at which open vacancies are filled, the value of the new job and the value of the vacancy, which is lost due to the filling of the job. Since there is free entry on the market, $V^{s}$ will be equal to zero in equilibrium. Solving for the value of a newly filled job yields

$$
J^{s}\left(\varepsilon_{u}\right)=\frac{c}{\phi\left(\theta^{s}\right)} .
$$

Hence, the value of a new job equals the expected search costs till a vacant position is filled, which implies that the value of a new job increases if the labor market becomes tighter. Turning to the worker, denote the value of having a job with an idiosyncratic productivity of $\varepsilon$ with $W^{s}(\varepsilon)$ and the value of unemployment with $U^{s}$. Hence, the total value of a match is

$$
S^{s}(\varepsilon)=J^{s}(\varepsilon)+W^{s}(\varepsilon)-U^{s}
$$

Assume further that as the result of some bargaining process the match surplus $S^{s}(\varepsilon)$ is divided up between the firm and the worker at the rate $\beta$ to $1-\beta$, $0<\beta<1$ :

$$
\begin{aligned}
J^{s}(\varepsilon) & =(1-\beta) S^{s}(\varepsilon), \\
W^{s}(\varepsilon)-U^{s} & =\beta S^{s}(\varepsilon) .
\end{aligned}
$$

A strong position of workers (or unions) in wage negotiations would correspond to a high value of $\beta$. Evidently, the value of the match cannot become negative (at least in this setting without firing costs). If it did, workers would quit into unemployment, whereas firms would disband the job. 
In a similar manner as in (1), the three value functions $J^{s}(\varepsilon), W^{s}(\varepsilon)$ and $U^{s}$ can be implicitly defined by

$$
\begin{aligned}
r J^{s}(\varepsilon) & =p+\sigma \varepsilon-w^{s}(\varepsilon)+\lambda(1-\beta) \int\left\{\max \left[S^{s}(x), 0\right]-S^{s}(\varepsilon)\right\} d F(x) \\
r W^{s}(\varepsilon) & =w^{s}(\varepsilon)+\lambda \beta \int\left\{\max \left[S^{s}(x), 0\right]-S^{s}(\varepsilon)\right\} d F(x) \\
r U^{s} & =b+\phi\left(\theta^{s}\right) \theta^{s}\left[W^{s}\left(\varepsilon_{u}\right)-U^{s}\right]
\end{aligned}
$$

The terms $p+\sigma \varepsilon-w^{s}(\varepsilon)$ and $w^{s}(\varepsilon)$ in equations (6) and (7) are the returns per period for the firm and the worker, respectively. In addition, the integrals capture the expected future change in the value of the job due to changes in the job-specific productivity, taking into account that the total value of the job must not fall below 0 if the job is to persist.

The second part of (8) is the expected return to searching for a job, since $\theta^{s} \phi\left(\theta^{s}\right)=$ $\frac{m\left(v^{s}, u^{s}\right)}{u^{s}}=m\left(\theta^{s}, 1\right)$ is the rate at which workers leave unemployment for a new job. Contrary to the function $\phi(\cdot)$ itself, $\theta^{s} \phi\left(\theta^{s}\right)$ increase with $\theta^{s}$, since an increase in labor market tightness makes it more likely to become employed. Note that for the worker the initial value of being matched to a vacancy is $W^{s}\left(\varepsilon_{u}\right)-U^{s}$ by the assumption that new jobs are equipped with the most productive technology.

Using $V^{s}=0$ and equations (2), (4) and (5) it may be shown that in equilibrium the return to searching for a job equals $\frac{\beta c}{1-\beta} \theta^{s}$.

The equations above can be solved for the total value of a match: ${ }^{7}$

$$
\begin{aligned}
(r+\lambda) S^{s}(\varepsilon) & =p+\sigma \varepsilon-b+\lambda \int_{\varepsilon_{d}^{s}}^{\varepsilon_{u}} S^{q^{\prime}}(x)[1-F(x)] d x-\beta \theta^{s} \phi\left(\theta^{s}\right) S^{s}\left(\varepsilon_{u}\right)(9) \\
& =p+\sigma \varepsilon-b+\frac{\sigma \lambda}{r+\lambda} \int_{\varepsilon_{d}^{s}}^{\varepsilon_{u}}[1-F(x)] d x-\frac{\beta c}{1-\beta} \theta^{s}
\end{aligned}
$$

A job is destroyed if the total value becomes negative due to a shock to the job-specific component of productivity. Considering the knife-edge case where the total value of the match equals zero leads to an implicit definition of the respective threshold $\varepsilon_{d}^{s}$,

$$
p+\sigma \varepsilon_{d}^{s}=b+\frac{\beta c}{1-\beta} \theta^{s}-\frac{\sigma \lambda}{r+\lambda} \int_{\varepsilon_{d}^{s}}^{\varepsilon_{u}}[1-F(x)] d x .
$$

\footnotetext{
${ }^{7}$ Adding (6) and (7), subtracting (8) and taking into account (3) leads to an equation of the form $r S^{s}(\varepsilon)=\ldots$ Making use of (5) yields equation (9). From there, integration by parts together with (2) and (4) finally leads to (10).
} 
The left hand side is the threshold value of total productivity below which a job is destroyed. The first two expressions on the right hand side, i.e. unemployment benefits and the return to searching for a job, are the opportunity costs of employment. This is the minimum wage the firm has to pay in order to keep the worker from quitting. The last expression measures the return to waiting for possible improvements in job-specific productivity and may therefore be called the option value of keeping the job. It enters the equation with a minus sign, meaning that at the threshold productivity it is profitable for the firm to pay a worker more than he produces. ${ }^{8}$ The reason why it does not pay to destroy the job as long as the losses are not too large is that the alternative option, i.e. destroying the job and posting a vacancy, incurs costs as well since the vacancy won't be filled instantaneously.

In order to analyze the dependence of $\varepsilon_{d}^{s}$ on the exogenous parameters, (11) has to be differentiated accordingly. Changes in the parameters are likely to affect labor market tightness $\theta^{s}$ as well, but this additional channel will be neglected for the moment in order to isolate the effect on job destruction and since taking account of the reaction in $\theta^{s}$ requires an equation describing job creation. Therefore, the following results are derived under the assumption $d \theta^{s}=0$. Note that a decline in $\varepsilon_{d}^{s}$ corresponds to an increase in job security, since it takes on average longer before the job is destroyed.

- An increase in $p-b$ lowers $\varepsilon_{d}^{s}$. A rise in global productivity $p$ makes more jobs with a lower job-specific productivity still profitable, whereas a reduction of $b$ reduces wages, with the same effect on jobs with a low productivity. ${ }^{9}$

- An increase in $\beta$ raises $\varepsilon_{d}^{s}$ (see the appendix), ${ }^{10}$ since the profit share of the firm has declined. Besides reducing the present value of future improvements in productivity, this also requires higher wages, because the workers'

\footnotetext{
${ }^{8}$ This can also be seen by plugging $\varepsilon_{d}^{s}$ into (6). Since $J^{s}\left(\varepsilon_{d}^{s}\right)=S^{s}\left(\varepsilon_{d}^{s}\right)=0$ and $\int \max \left\{S^{s}(x), 0\right\} d F(x)>0$, it follows that $p+\sigma \varepsilon_{d}^{s}<w^{s}\left(\varepsilon_{d}^{s}\right)$.

${ }^{9} \mathrm{~A}$ similar result can be found in Bentolila and Bertola (1990), where a band of inaction in the adjustment of labor demand is derived that depends on the level of product demand. Increases in demand also increase the width of the band.

${ }^{10}$ Contrary to most of the other effects, changes in $\beta$ and $c$ are not explicitly considered in Mortensen and Pissarides (1994) (they just look at the compound term $\frac{\beta c}{1-\beta}$ ). Therefore the claimed dependencies are derived in the appendix.
} 
expected return to searching has risen.

For similar reasons, an increase in $c$ raises $\varepsilon_{d}^{s}$. Equation (2) shows that for a given value of $\theta^{s}$, new jobs will be more profitable. Therefore, unprofitable jobs are more likely to be destroyed. Note that especially this result depends very much on the ceteris paribus assumption $d \theta^{s}=0$, since higher costs of maintaining a vacancy should have strong effects on the provision of new jobs, too.

- A higher value of $\lambda$ increases the option value of not destroying a job, since improvements in productivity become more likely, or, as Garibaldi (1997) puts it, shocks are less persistent. ${ }^{11}$ Therefore $\varepsilon_{d}^{s}$ decreases.

- Increasing the discount rate $r$ lowers the option value of keeping the job since the present value of future profits sinks. Therefore, the threshold $\varepsilon_{d}^{s}$ rises.

- Differentiating (11) with respect to $\sigma$ yields

$$
\sigma \frac{\partial \varepsilon_{d}^{s}}{\partial \sigma}=\frac{r+\lambda}{\sigma\left(r+\lambda F\left(\varepsilon_{d}^{s}\right)\right)}\left(p-b-\frac{\beta c}{1-\beta} \theta^{s}\right) .
$$

This shows that the effect of a rise in the dispersion parameter $\sigma$ depends on the relative size of the global productivity and the opportunity costs of employment. The derivative is positive if $p \geq b+\frac{\beta c}{1-\beta} \theta^{s} .{ }^{12}$

As already stated before, the previous analysis has neglected the endogeneity of the labor market tightness variable $\theta^{s}$. From equations (2), (4) and (10) one obtains the following equation

$$
\phi\left(\theta^{s}\right)=\frac{c}{1-\beta} \frac{r+\lambda}{\sigma\left(\varepsilon_{u}-\varepsilon_{d}^{s}\right)}
$$

that gives an implicit description of the creation of new jobs. Equations (11) and (13) jointly determine $\varepsilon_{d}^{s}$ and $\theta^{s}$. Note that in (11) the relation between the two variables is positive, since an increase in labor market tightness $\theta^{s}$ raises wages via the opportunity cost of employment, so that firms destroy jobs at higher values of $\varepsilon$ than before. Contrary to that, there is an inverse relationship in (13),

\footnotetext{
${ }^{11}$ If the situation deteriorates even more, the job can still be destroyed. As long as the current losses mentioned above are not too large, the returns to waiting should outweigh the costs.

${ }^{12}$ In that case, it follows from (11) that $\varepsilon_{d}^{s}<0=E(\varepsilon)$.
} 
meaning that a lower life-expectancy of existing jobs due to an increase in $\varepsilon_{d}^{s}$ leads to a reduction in the supply of vacancies (remember that $\phi\left(\theta^{q}\right)<0$ ).

Equations (11) and (13) can be differentiated with respect to the exogenous parameters already considered above. Determining the signs of the derivatives yields the following results:

- An increase in $p-b$ lowers $\varepsilon_{d}^{s}$ and raises $\theta^{s}$. The initial effect on $\varepsilon_{d}^{s}$ works as before. In addition, due to the higher productivity of new jobs firms try to create more of them which makes the labor market tighter. This improves the position of the workers and raises wages, which tends to counteract the initial effect on $\varepsilon_{d}^{s}$, but the net effect on the threshold productivity level is still negative.

- The reaction of $\varepsilon_{d}^{s}$ to an increase in $\beta$, labor's share of the total match value, is ambiguous, whereas $\theta^{s}$ declines unambiguously. This decline is also the reason why the the sign of $\partial \varepsilon_{d}^{s} / \partial \beta$ can no longer be determined uniquely. The lower labor market tightness reduces the value of unemployment (and the opportunity cost of employment), thereby raising the value of the match that can be split between the firm and the worker. If this effect is strong enough, $\varepsilon_{d}^{s}$ may even decline. In the appendix it is shown that $\partial \varepsilon_{d}^{s} / \partial \beta \lessgtr 0$ iff $\beta \lessgtr \eta$ (note that in general $\eta$ depends again on $\theta^{s}$ ).

An increase in $c$ lowers $\varepsilon_{d}^{s}$ as well as $\theta^{s}$. In this case, the decline in the opportunity cost of employment is strong enough to reverse the sign of $\partial \varepsilon_{d}^{s} / \partial c$ compared to the analysis for $d \theta^{s}=0$.

- A higher value of $\lambda$ leads to a reduction in $\varepsilon_{d}^{s}$ as well as $\theta^{s}$. A possible explanation for the latter effect could be that new jobs lose their high initial productivity more quickly, making it less attractive to create them in the first place.

- As with an increase in $\beta$, a higher discount rate $r$ has an ambiguous effect on $\varepsilon_{d}^{s}$, but lowers $\theta^{s}$ unambiguously.

- Labor market tightness is an increasing function of $\sigma$. The threshold $\varepsilon_{d}^{s}$ increases, too, if the condition $p \geq b$ holds. Taking into account the change in $\theta^{s}$ reinforces the effect on $\varepsilon_{d}^{s}$, since now a weaker condition is sufficient to obtain a positive sign of $\partial \varepsilon_{d}^{s} / \partial \sigma$. 
Nothing has been said up to now about unemployment or the absolute number of vacancies. In order to determine the equilibrium values of $v^{s}$ and $u^{s}$, a third condition is needed. The labor market will be in equilibrium if flows into unemployment equal outflows. Normalizing the total number of skilled workers to 1 and noting that workers will be dismissed if their job is hit by a shock that changes the idiosyncratic productivity to a value below $\varepsilon_{d}^{s}$, the condition for a flow-equilibrium may be written as

$$
m\left(v^{s}, u^{s}\right)=\left(1-u^{s}\right) \lambda F\left(\varepsilon_{d}^{s}\right)
$$

Solving for the number of unemployed $u^{s}$ (which, due to our normalization, equals the unemployment rate) yields

$$
u^{s}=\frac{\lambda F\left(\varepsilon_{d}^{s}\right)}{\lambda F\left(\varepsilon_{d}^{s}\right)+m\left(v^{s} / u^{s}, 1\right)} .
$$

Equation (15) defines a Beveridge-Curve (BV) with the usual negative slope. In the $u^{s}-v^{s}$-space, the equilibrium value of labor market tightness derived from equations (11) and (13) defines a straight line through the origin with a slope of $\theta^{s}$ (SS). The intersection of the two curves determines the equilibrium values of $u^{s}$ and $v^{s}$, compare figure 1 . Using the results on the effects of parameter changes

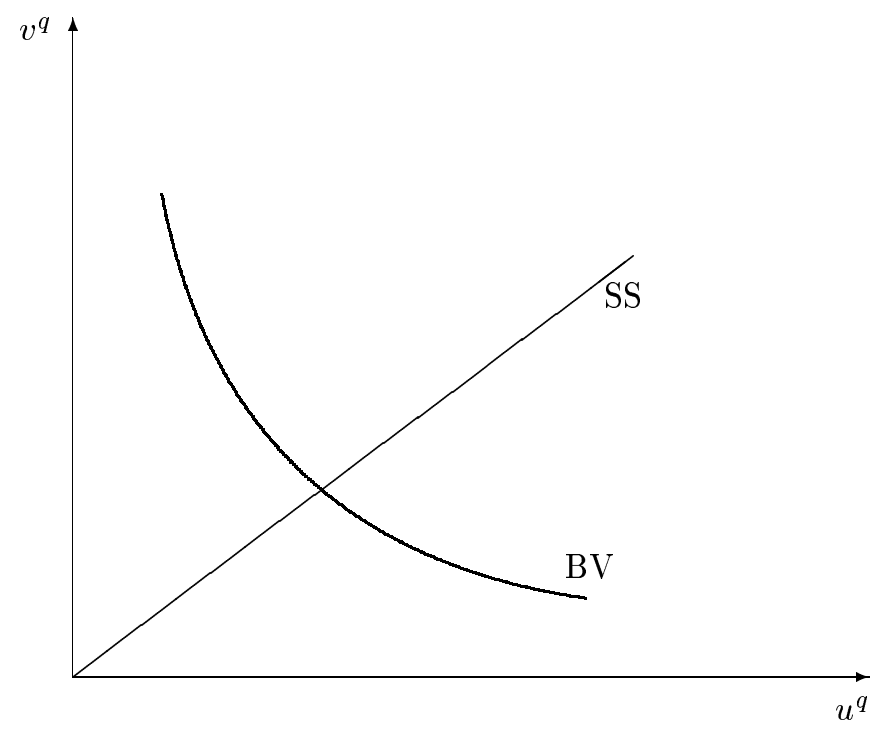

Figure 1: Beveridge- and SV-curve

on $\varepsilon_{d}^{s}$ and $\theta^{s}$ from above allows a graphical analysis of the effects on $u^{s}$ and $v^{s}$. 
An increase in $\varepsilon_{d}^{s}$ shifts the BV-curve out (see equation (14)). A higher value of $\theta^{s}$ turns the SS-curve upwards.

An increase in $p-b$, raising $\theta^{s}$ and lowering $\varepsilon_{d}^{s}$, turns the SS-curve up and shifts the BV-curve in. Unemployment declines, but the effect on the number of vacancies is unclear. Raising $\lambda$ turns the SS-curve down. As regards the BVcurve, two effects have to be distinguished, since $\lambda$ enters (15) directly and also via $\varepsilon_{d}^{s}$. The direct effect shifts the BV-curve out, while the indirect effect works in the opposite direction. Unemployment will rise (with ambiguous effects on $v^{s}$ ), if the first effect dominates. If the indirect effect is stronger, vacancies will decline, but $u^{s}$ may rise or fall. ${ }^{13}$

If vacancies become more expensive (i.e. $c$ rises), the SS-curve turns down and the BV-curve shifts in. This leads to a decline in $v^{s}$, as one would expect, but the effect on unemployment is again indeterminate (it will rise if the decrease in labor market tightness is very strong relative to the change in $\varepsilon_{d}^{s}$ ). Finally, suppose the condition $p \geq b$ holds. In this case, a higher value of $\sigma$ turns the SS-curve up and shifts the BV-curve out, so that the number of vacancies rises. Again, the effect on unemployment is unclear.

\subsection{The labor market for the low-skilled}

Both labor market segments are structurally similar, so that I will resort to results from the previous subsection where possible and rather elaborate on the differences of the two segments.

Equations (1) - (5), (7) and (8) also hold for the low-skill segment analogously, i.e. l-superscripts have to be substituted for $s$-superscripts. However, due to the lower productivity, equation (6) needs to be (slightly) modified into

$$
r J^{l}(\varepsilon)=p+\sigma(\varepsilon-\rho)-w^{l}(\varepsilon)+\lambda(1-\beta) \int\left\{\max \left[S^{l}(x), 0\right]-S^{l}(\varepsilon)\right\} d F(x) .
$$

Following the same steps as before, one arrives at two equations that define the

\footnotetext{
${ }^{13}$ Mortensen and Pissarides (1994) note that the indirect effect of $\lambda$ works through the integral term in equation (11). This term vanishes for $\sigma \rightarrow 0$ and becomes independent of $\lambda$ for $r \rightarrow 0$, so that in both cases the indirect effect will vanish.
} 
total value of a match and the threshold $\varepsilon_{d}^{l}$ in the low-skill segment,

$$
\begin{aligned}
(r+\lambda) S^{l}(\varepsilon) & =p+\sigma(\varepsilon-\rho)-b+\frac{\sigma \lambda}{r+\lambda} \int_{\varepsilon_{d}^{l}}^{\varepsilon_{u}}[1-F(x)] d x-\frac{\beta c}{1-\beta} \theta^{l}, \\
p+\sigma\left(\varepsilon_{d}^{l}-\rho\right) & =b+\frac{\beta c}{1-\beta} \theta^{l}-\frac{\sigma \lambda}{r+\lambda} \int_{\varepsilon_{d}^{l}}^{\varepsilon_{u}}[1-F(x)] d x .
\end{aligned}
$$

Defining a new variable $\tilde{p}:=p-\sigma \rho$ shows that the results of the analysis performed in subsection 2.2, holding labor market tightness fixed, apply here as well. Increases in $p-b$ and $\lambda$ lower $\varepsilon_{d}^{l}$, whereas increases in $r, \beta$ and $c$ lead to a higher threshold value. In addition, the jobs of the low-skilled will be destroyed more quickly, $\varepsilon_{d}^{l} \geq \varepsilon_{d}^{s}$, since $\tilde{p} \leq p$. In the appendix it is shown that the threshold reacts more than proportionally to a change in $\rho, \partial \varepsilon_{d}^{l} / \partial \rho>1$. Additional insights can be gained by looking at the cross-derivatives of $\varepsilon_{d}^{l}$ with respect to $\rho$ and one of the other parameters. The effect of the productivity differential on the threshold increases with global productivity, $\partial^{2} \varepsilon_{d}^{l} / \partial \rho \partial(p-b)>0$. Put the other way round, i.e. reading the partial derivative as $\partial\left(\frac{\partial \varepsilon_{d}^{l}}{\partial(p-b)}\right) / \partial \rho$, the larger the productivity differential, the less do the low-skilled profit from increases in global productivity. ${ }^{14}$ The sign of $\partial^{2} \varepsilon_{d}^{l} / \partial \rho \partial \lambda$ is positive as well, which may be interpreted in a similar manner ( $\lambda$ could e.g be interpreted as measuring the degree or speed of technological change). The cross-derivatives with respect to $\beta$ and $r$ are negative, meaning that the detrimental effects of increases in those parameters become less important with higher values of $\rho$.

As regards changes in $\sigma$, the term $-\sigma \rho$ has to be taken into account, but after some manipulations one arrives at

$$
\sigma \frac{\partial \varepsilon_{d}^{l}}{\partial \sigma}=\frac{r+\lambda}{\sigma\left(r+\lambda F\left(\varepsilon_{d}^{l}\right)\right)}\left(p-b-\frac{\beta c}{1-\beta} \theta^{l}\right),
$$

which is completely analogous to (12). Therefore, a larger spread in idiosyncratic shocks raises the threshold if $p \geq b+\frac{\beta c}{1-\beta} \theta^{l}$. Finally, the cross derivative $\partial^{2} \varepsilon_{d}^{l} / \partial \rho \partial \sigma$ is negative. A first tentative conclusion from this analysis would therefore be that the low-skilled profit less from beneficial changes in the economy, but that the effect of detrimental changes is smaller, too.

In order to take account of the endogeneity of $\theta^{l}$, an equation that describes the creation of jobs is needed. Following the steps that led to (13), the parameter $\rho$

\footnotetext{
${ }^{14}$ Remember that $\partial \varepsilon_{d}^{l} / \partial(p-b)<0$ and that a decline in the threshold means an increase in job security.
} 
drops out and one arrives at (see the appendix)

$$
\phi\left(\theta^{l}\right)=\frac{c}{1-\beta} \frac{r+\lambda}{\sigma\left(\varepsilon_{u}-\varepsilon_{d}^{l}\right)} .
$$

As before, equations (18) and (20) jointly determine the equilibrium values of $\varepsilon_{d}^{l}$ and $\theta^{l}$. The results of subsection 2.2 on the dependence of these two variables on $p-b, \beta, c, r$ and $\lambda$ continue to hold. Since a decrease in $p-b$ raises $\varepsilon_{d}^{l}$ and lowers $\theta^{l}$, it follows immediately that the low-skilled have a higher threshold value and a more unfavorable ratio of vacancies to the number of unemployed. In other words, the life span of a low-skill job is shorter and the labor market segment for the low skilled is less tight even with flexible wages. ${ }^{15}$

The derivative $\partial \varepsilon_{d}^{l} / \partial \sigma$ is positive for $p \geq b$ as in the previous subsection, but the sign of $\partial \theta^{l} / \partial \sigma$ becomes unclear. In the appendix it is shown that the derivative will only be positive if the productivity differential is not too large. To be more specific, a sufficient condition for this is $E\left(\varepsilon \mid \varepsilon \geq \varepsilon_{d}^{l}\right)>\rho$, i.e. the average idiosyncratic productivity among the low-skilled should not be negative.

An increase in the productivity differential $\rho$, e.g. due to skill-biased technological change, raises the threshold and reduces labor market tightness. The cross derivative $\partial^{2} \varepsilon_{d}^{l} / \partial \rho \partial(p-b)$ can now become negative, but it will remain positive (as in the analysis for a fixed $\theta^{l}$ ) if $\phi^{\prime \prime}\left(\theta^{l}\right)$ is large in absolute numbers, so that $\phi(\cdot)$ is strongly convex. ${ }^{16}$

The flow equilibrium in the labor market segment for the low-skilled implies again a Beveridge-curve which is described by

$$
u^{l}=\frac{\lambda F\left(\varepsilon_{d}^{l}\right)}{\lambda F\left(\varepsilon_{d}^{l}\right)+m\left(v^{l} / u^{l}, 1\right)} .
$$

Apart from the slightly stronger conditions required for the derivatives with respect to $\sigma$, the effects of changes in $p-b, \beta, c, \lambda, r$ and $\beta$ on the curves are qualitatively the same as in the previous subsection. Skill biased technological change in the form of a rise in $\rho$ turns the SS-curve down and shifts the BV-curve

\footnotetext{
${ }^{15}$ Note also from (12) and (19) (i.e. when holding $\theta^{\nu}$ fixed) that because of $\theta^{l}<\theta^{s}$ the condition for $\partial \varepsilon_{d}^{l} / \partial \sigma>0$ is automatically fulfilled if $\partial \varepsilon_{d}^{s} / \partial \sigma>0$, meaning that when a higher dispersion of job-specific shocks leads to a shorter lifespan of jobs for the skilled, then the low-skilled will loose their jobs more quickly all the more so.

${ }^{16}$ In the special case of a Cobb-Douglas matching function the sign of the cross derivative still remains uncertain.
} 
out, so that unemployment among the low-skilled rises. The effect on the number of vacancies is ambiguous.

One of the basic assumptions in subsection 2.1 was that workers only apply for jobs on their 'own' market. Suppose that this assumption is somewhat relaxed so that as in Coe and Snower (1997) the skilled can also apply for low-skill jobs, whereas the reverse is still not possible because the low-skilled cannot perform the tasks pertaining to a job designed for the skilled. Assume further that due to technological constraints the skilled workers employed for low-skill jobs have the same productivity as the low-skilled, which implies that they will get the wage of a low-skilled worker and have the same probability of finding such a job. Simultaneous applications on both markets are forbidden. It was shown that the labor market for the skilled is tighter, $\theta^{s}>\theta^{l}$. Therefore, the rate at which an unemployed worker finds a new job is lower in the market segment for the lowskilled. Because of $\phi^{\prime}(\cdot)<0$, equations (2), (4), (5) and their analogues for the low-skilled, the value of a match for the worker is lower in the low-skill segment. This means that the return to searching for a job is higher in the market segment for the skilled, and a skilled worker will not search for low-skill jobs even if he has the possibility to do so. To put it differently, in this model the higher relative scarcity of the skilled prevents them from competing with the low-skilled for jobs.

\section{Introducing firing costs}

Up to now, it was assumed that an unproductive job could be destroyed costlessly. In practice, firing workers most likely involves explicit or implicit costs like severance payments, attorney's fees, mandatory notification periods during which the worker has to be kept employed or dissatisfaction among the remaining workforce. This section sketches the consequences of incorporating these costs in the model. As before, the analysis starts with the labor segment for the skilled and discusses particularities of the unskilled after that.

Assume that laying off a worker causes firing costs $k$ per job. For simplicity, firing costs are assumed to be independent of the skill level of the worker (as it was also assumed with the unemployment benefit $b$ ). Let the value of $k$ be such 
that $S^{l}\left(\varepsilon_{u}\right)>0$ (and therefore also $S^{s}\left(\varepsilon_{u}\right)$ ), since otherwise no new jobs would be created and all firms would leave the market. Firing costs are not paid to the worker: they are either given to a third party or completely lost. ${ }^{17}$ The dismissal is costless from the perspective of the worker, so that the firm cannot shift $k$ or part of it to the worker. ${ }^{18}$

Under these assumptions, a job will be destroyed if its value sinks below the firing costs, i.e. if

$$
J^{s}(\varepsilon)<-k
$$

since in the interval $0>J^{s}(\varepsilon) \geq-k$ the firm prefers keeping the job to paying $-k$.

Equations (1) and (2) are unchanged. However, since $k$ cannot be shifted to the worker, the splitting rules (4) and (5) have to be modified. If the total value of the match gets negative, it will be borne entirely by the firm. On the other hand, the value of the match to the worker is bounded from below by zero. Hence, the new splitting rules are

$$
\begin{aligned}
J^{s}(\varepsilon) & = \begin{cases}(1-\beta) S^{s}(\varepsilon), & \text { if } S^{s}(\varepsilon) \geq 0 \\
S^{s}(\varepsilon) & \text { if }-k \leq S^{s}(\varepsilon)<0 \\
-k & \text { if } S^{s}(\varepsilon)<-k\end{cases} \\
& =\max \left\{\min \left\{(1-\beta) S^{s}(\varepsilon), S^{s}(\varepsilon)\right\},-k\right\},
\end{aligned}
$$

and

$$
W^{s}(\varepsilon)-U^{s}=\max \left\{\beta S^{s}(\varepsilon), 0\right\}
$$

These two equations show that $S^{s}(\varepsilon)$, the total value of the match, is still a simple sum of its components $J^{s}(\varepsilon)$ and $W^{s}(\varepsilon)-U^{s}$. Therefore, (3) still holds, but the underlying partitioning of the total value has changed.

\footnotetext{
${ }^{17}$ One could conceive schemes under which the firm has to pay fines to the state or employment programs from which those that are actually dismissed derive only negligible utility.

${ }^{18}$ An alternative would be to define costs $\tilde{k}$ relative to the total value of the match $S^{s}(\varepsilon)$ (and not relative to the share of the firm, $J^{s}(\varepsilon)$, as it is done here). In such a framework, a job would be dissolved if we had $S^{s}(\varepsilon)<-\tilde{k} \Leftrightarrow J^{s}(\varepsilon)<-(1-\beta) \tilde{k}$. From this perspective, $\tilde{k}$ describes rather 'quasi-firing-costs', since only a fraction $1-\beta$ is borne by the firm. This in turn implies that for a certain interval of the idiosyncratic productivity, the value of the match to the worker would be negative, i.e. $W^{s}(\varepsilon)-U<0$. For this to be an equilibrium, one would have to assume some frictions that prevent the worker from quitting the job voluntarily.

Technically, this approach would be easier, since the splitting rules (4) and (5) would continue to hold, which is not the case in the specification chosen in this section.
} 
In addition, firing costs have to be taken into account in the definitions of $J^{s}(\varepsilon)$ and $W^{s}(\varepsilon)-U^{s}$. As regards the option value of not destroying a job, one now has to differentiate whether it is still positive or already less than zero. Denote by $\mathcal{S}_{+}^{s}:=\left\{\varepsilon \mid S^{s}(\varepsilon) \geq 0\right\}$ the set of $\varepsilon$-schocks for which the total value of the match is non-negative (so that the 'old' splitting rules would still apply) and let $\mathcal{S}_{-}:=\overline{\mathcal{S}_{+}}=\mathbb{R} \backslash \mathcal{S}_{+}$be the complement to this set. Using this notation and splitting rule (23), the option value to the firm is

$$
\begin{aligned}
& -\lambda \int_{\mathcal{S}_{+}}\left[(1-\beta) S^{s}(x)-(1-\beta) S^{s}(\varepsilon)\right] d F(x)+\lambda \int_{\mathcal{S}_{-}}\left[\max \left\{S^{s}(x),-k\right\}-(1-\right. \\
& \left.\quad \beta) S^{s}(\varepsilon)\right] d F(x) \text { if } \varepsilon \in \mathcal{S}_{+} \text {, and } \\
& \text { - } \lambda \int_{\mathcal{S}_{+}}\left[(1-\beta) S^{s}(x)-S^{s}(\varepsilon)\right] d F(x)+\lambda \int_{\mathcal{S}_{-}}\left[\max \left\{S^{s}(x),-k\right\}-S^{s}(\varepsilon)\right] d F(x), \\
& \quad \text { if } \varepsilon \in \mathcal{S}_{-} .
\end{aligned}
$$

Noting that $k$ cannot be shifted to the worker, his share of the option value is

$$
\begin{aligned}
& \text { - } \lambda \int_{\mathcal{S}_{+}}\left[\beta S^{s}(x)-\beta S^{s}(x)\right] d F(x)+\lambda \int_{\mathcal{S}_{-}}-\beta S^{s}(\varepsilon) d F(x), \text { if } \varepsilon \in \mathcal{S}_{+} \text {, and } \\
& \text { - } \lambda \int_{\mathcal{S}_{+}} \beta S^{s}(x) d F(x) \text {, if } \varepsilon \in \mathcal{S}_{-} .
\end{aligned}
$$

The value of unemployment, $U^{s}$, is independent of $\varepsilon$, so that a fall differentiation is unnecessary.

As before, one has to solve for $S^{s}(\varepsilon)$, from which an equation for $\varepsilon_{d}^{s}$ can be derived. Let $1_{\{A\}}$ be the indicator function for event $A$, i.e. $1_{\{A\}}=1$ if $A$ happens and $1_{\{A\}}=0$ otherwise. By means of this function, the two events $\varepsilon \in \mathcal{S}_{+}^{s}$ and $\varepsilon \in \mathcal{S}_{-}^{s}$ can be expressed within one equation, so that $S^{s}(\varepsilon)=J^{s}(\varepsilon)+W^{s}(\varepsilon)-U^{s}$ may be written as

$$
r S^{s}(\varepsilon)=p+\sigma \varepsilon-b-\beta \theta^{s} \phi\left(\theta^{s}\right) S^{s}\left(\varepsilon_{u}\right)+1_{\left\{\varepsilon \in \mathcal{S}_{+}\right\}} T_{+}^{s}+1_{\left\{\varepsilon \in \mathcal{S}_{-}\right\}} T_{-}^{s}
$$

with

$$
\begin{aligned}
T_{+}^{s} & =\lambda \int_{\mathcal{S}_{+}}\left[S^{s}(x)-S^{s}(\varepsilon)\right] d F(x)+\lambda \int_{\mathcal{S}_{-}}\left[\max \left\{S^{s}(x),-k\right\}-S^{s}(\varepsilon)\right] d F(x) \\
& =-\lambda S^{s}(\varepsilon)+\lambda \int_{\mathcal{S}_{+}} S^{s}(\varepsilon) d F(x)+\lambda \int_{\mathcal{S}_{-}} \max \left\{S^{s}(x),-k\right\} d F(x) \\
& =-\lambda S^{s}(\varepsilon)+\lambda \int_{\varepsilon_{d}^{s}}^{\varepsilon_{u}} S^{s}(x) d F(x)-\lambda k F\left(\varepsilon_{d}^{s}\right)
\end{aligned}
$$


and similarly

$$
\begin{aligned}
T_{-}^{s} & =\lambda \int_{\mathcal{S}_{+}}\left[S^{s}(x)-S^{s}(\varepsilon)\right] d F(x)+\lambda \int_{S_{-}}\left[\max \left\{S^{s}(x),-k\right\}-S^{s}(\varepsilon)\right] d F(x) \\
& =-\lambda S^{s}(\varepsilon)+\lambda \int_{\varepsilon_{d}^{s}}^{\varepsilon_{u}} S^{s}(x) d F(x)-\lambda k F\left(\varepsilon_{d}^{s}\right)
\end{aligned}
$$

Since the addends are the same in both cases, the fall differentiation can be dropped, and (25) may be rewritten as

$$
\begin{aligned}
(r+\lambda) S^{s}(\varepsilon)=p+\sigma \varepsilon-b-\beta \theta^{s} \phi\left(\theta^{s}\right) & S^{s}\left(\varepsilon_{u}\right) \\
+\lambda & {\left[\int_{\varepsilon_{d}^{s}}^{\varepsilon_{u}} S^{s}(x) d F(x)-k F\left(\varepsilon_{d}^{s}\right)\right] . }
\end{aligned}
$$

Note that equation (9) is a special case of (28) for $k=0$.

Integration by parts (see the appendix for details) leads to a rather familiar equation determining the value of a total match in the presence of firing costs,

$$
(r+\lambda) S^{s}(\varepsilon)=p+\sigma \varepsilon-b-\frac{\beta c}{1-\beta} \theta^{s}+\frac{\sigma \lambda}{r+\lambda} \int_{\varepsilon_{d}^{s}}^{\varepsilon_{u}}[1-F(x)] d x-\lambda k
$$

The only visible difference between (29) and (10) is the term $-\lambda k$, which captures something like the expected firing cost (the more likely changes in the job-specific productivity become, the earlier firing costs will have to be paid, thereby lowering the value of the match). What does the endogenous threshold $\varepsilon_{d}^{s}$ look like? Since the value of the match equals the firing costs at the point where the firm is indifferent between destroying and maintaining the job, $S^{s}\left(\varepsilon_{d}^{s}\right)=-k$, one obtains from (29)

$$
p+\sigma \varepsilon_{d}^{s}=b+\frac{\beta c}{1-\beta} \theta^{s}-\frac{\sigma \lambda}{r+\lambda} \int_{\varepsilon_{d}^{s}}^{\varepsilon_{u}}[1-F(x)] d x-r k .
$$

The equation shows that firing costs tend to raise the option value of keeping the job. This is because firms, trying to avoid having to pay $k$, will accept lower levels of job-specific productivity in the hope that future shocks will make the job profitable again. Consequently, the derivative of $\varepsilon_{d}^{s}$ with respect to $k$ (holding $\theta^{s}$ constant) is negative, and workers can on average keep their jobs longer. Insofar firing costs are an effective instrument for increasing job security.

The sign of the derivative $\partial \varepsilon_{d}^{s} / \partial r$ is now ambiguous: On the one hand, future returns are discounted more heavily, which lowers the option value of keeping the 
job and thus raises $\varepsilon_{d}^{s}$. On the other hand, more weight is put on firing $\operatorname{costs} k$, which lowers $\varepsilon_{d}^{s}$.

The condition for an increase in $\sigma$, the variance of job-specific shocks, to raise $\varepsilon_{d}^{s}$ now changes to $p+r k>b+\frac{\beta c}{1-\beta} \theta^{s}$, which for a given value of $\theta^{s}$ is weaker than before. In addition, the cross-derivative of $\varepsilon_{d}^{s}$ with respect to $\sigma$ and $k$ is positive. Reading the cross-derivative as $\partial\left(\frac{\partial \varepsilon_{d}^{s}}{\partial k}\right) / \partial \sigma$, this means that the effect of $k$ on job security is lower if the idiosyncratic productivity is more volatile (measured by its standard deviation $\sigma$ ). The signs of the derivatives with respect to $p-b$, $c$ and $\lambda$ do not change, since the expression capturing the firing costs enters (30) linearly, so that it drops out during the differentiation.

In order to analyze the effects of firing costs on $\varepsilon_{d}^{s}$ taking into account the simultaneous reaction of $\theta^{s}$, a new equation that determines $\theta^{s}$ is needed. As in subsection 2.2, one obtains from equations (1)-(3) and (23)

$$
\phi\left(\theta^{s}\right)=\frac{c}{(1-\beta) S^{s}\left(\varepsilon_{u}\right)} .
$$

From equation (29) one gets

$$
\begin{aligned}
S^{s}(\varepsilon)-S^{s}\left(\varepsilon_{d}^{s}\right) & =\frac{\sigma\left(\varepsilon-\varepsilon_{d}^{s}\right)}{r+\lambda} \\
\Leftrightarrow \quad S^{s}(\varepsilon) & =\frac{\sigma\left(\varepsilon-\varepsilon_{d}^{s}\right)}{r+\lambda}-k .
\end{aligned}
$$

Writing (33) for $\varepsilon=\varepsilon_{u}$ and plugging it into (31) finally yields the desired equation that determines $\theta^{s}$,

$$
\phi\left(\theta^{s}\right)=\frac{c(r+\lambda)}{(1-\beta)\left[\sigma\left(\varepsilon_{u}-\varepsilon_{d}^{s}\right)-(r+\lambda) k\right]} .
$$

For a given value of $\varepsilon_{d}^{s}$, higher firing costs increase the right hand side, ${ }^{19}$ thereby reducing $\theta^{s}$. Differentiating (30) and (34) with respect to $k$ shows that qualitatively the results previously derived for $d \theta^{s}=0$ continue to hold: an increase in $k$ lowers $\varepsilon_{d}^{s}$, which means that job security increases. As one would expect, the derivative $\partial \theta^{s} / \partial k$ is negative, i.e. open jobs become relatively more scarce.

The change in equation (34) makes it more difficult to analyze the effects of changes in the other parameters, but it is still relatively straightforward to show that an increase in $p-b$ raises $\theta^{s}$ and lowers $\varepsilon_{d}^{s}$, as in the case where $k=0$.

\footnotetext{
${ }^{19}$ Values of $k$ such that the right hand side becomes negative are assumed away.
} 
In figure 1 on page 10, an increase in firing costs shifts the SS-curve down and the BV-curve in. The effect on unemployment is unclear, but vacancies unambiguously decline. ${ }^{20}$

The labor market segment for the low-skilled: With respect to the analysis for $d \theta^{l}=0$ no modifications in addition to those made in this section and subsection 2.3 are required. In particular, an increase in $\rho$ still raises $\varepsilon_{d}^{l}$. Furthermore, it may be shown that the cross-derivative $\partial\left(\frac{\partial \varepsilon_{d}^{l}}{\partial \rho}\right) / \partial k$ is positive. This means that the adverse effects of a higher productivity differential is a rising function in $k$, or put differently (i.e. writing the cross derivative as $\left.\partial\left(\frac{\partial \varepsilon_{d}^{l}}{\partial k}\right) / \partial \rho\right)$, firing costs protect the skilled more than the low-skilled.

Allowing $\theta^{l}$ to vary, it can be shown that as in the case $k=0$, an increase in $\rho$ raises $\varepsilon_{d}^{l}$ and lowers $\theta^{l}$, so that the low-skilled will be dismissed earlier and labor market tightness will decrease in their segment. In addition, under the assumption of a Cobb-Douglas matching function, the cross-derivative $\partial\left(\frac{\partial \varepsilon_{d}^{l}}{\partial k}\right) / \partial \rho$ will be positive as in the case of $d \theta^{l}=0$, so that the low-skilled still profit less from firing costs (see the appendix).

Since an increase in $k$ reduces $\theta^{\nu}, \nu=s, l$, the probability of finding a job which was defined as $\theta^{\nu} \phi\left(\theta^{\nu}\right)=m\left(v^{\nu} / u^{\nu}, 1\right)=m\left(\theta^{\nu}, 1\right)$ will go down as well. If there is a risk that workers lose some of their human capital or give up searching actively for a job, ${ }^{21}$ they will effectively drop out of the labor market even though they might still be counted as unemployed. If this effect were strong enough, firing costs could unambiguously increase unemployment. A similar result would hold if long-term unemployment among the skilled led to a deterioration of their abilities so that they would become low-skilled. Since the unemployment rate among the low-skilled is higher and since the total unemployment rate is an employment-weighted average of $u^{s}$ and $u^{l}$, higher firing costs would lead to more unemployment as well.

\footnotetext{
${ }^{20}$ The fact that firing costs do not necessarily increase unemployment is a feature of several models, see e.g. Bentolila and Bertola (1990) or Saint-Paul (1996b, chp. 6).

${ }^{21}$ For a model in this vein see Pissarides (1992).
} 


\section{Business Cycles}

In the present framework, business cycles can be interpreted as fluctuations in the aggregate productivity $p$. The effects of changes in $p$ have already been addressed in the previous sections, but all the analyses were exercises in comparative statics, since they only compared steady states. Put differently, firms and workers did not expect $p$ to change, and this is precisely the reason why business cycles have to be looked at separately.

In the presence of cycles, there is always a positive probability that the economic environment will change. As with idiosyncratic shocks, firms should take this into account when valuing existing and new jobs. Assume for simplicity that there are two values of global productivity, $\check{p}$ and $p$, with $\check{p}>p$. Hence, $\check{p}$ characterizes booms, and all variables pertaining to this state of the world will be marked with a check " " " as well. The transition between booms and recessions is governed by a Poisson process with parameter $\mu$. The rest of the section assumes the existence of firing costs. The scenario without firing costs that has been analyzed by Mortensen and Pissarides (1994) is a special case that can be obtained by setting $k=0$.

Incorporating these changes into the model requires the use of two versions of its equations, some of which are however structurally identical in both regimes. Equations (1)-(3), (23) and (24) for example look the same in booms and recessions, except for the fact that in the first case $\theta^{s}, V(\cdot), S(\cdot), J(\cdot), W(\cdot)$ and $U$ carry a " " " The equations for $J^{s}(\cdot), W^{s}(\cdot)$ and $U^{s}$ in section 3 , however, which define the value of a job as a function of current and future returns have to be modified in order to capture the additional possibility of a change in value due to a change in aggregate productivity.

In the case of $\breve{J}^{s}(\varepsilon)$ - the value of a job to a firm in times of a boom - the expected value of this change amounts to $\mu\left[J^{s}(\varepsilon)-\breve{J}^{s}(\varepsilon)\right]$ if the job is still alive after the downturn and $\mu\left[-k-\check{J}^{s}(\varepsilon)\right]$ if the job is destroyed because of the recession. Conversely, for a firm that already operates in a recession, the expected change in

value is $\mu\left[\breve{J}^{s}(\varepsilon)-J^{s}(\varepsilon)\right]$. A fall differentiation is not needed since a job that was not destroyed in the more hostile environment of a downturn will be profitable to keep in a boom all the more. More formally, the steady-state analysis showed that a rise in $p$ leads to a decline in $\varepsilon_{d}^{s}$, so that $\varepsilon \geq \varepsilon_{d}^{s}$ implies $\varepsilon \geq \breve{\varepsilon}_{d}^{s}$. Similar 
considerations can be made for the equations defining $\check{W}^{s}(\varepsilon), W^{s}(\varepsilon), \check{U}^{s}$ and $U^{s}$. Applying the same transformations as in section 3 yields equations that define $S^{s}(\varepsilon)$ und $\check{S}^{q}(\varepsilon)$ :

$$
\begin{gathered}
(r+\lambda+\mu) S^{s}(\varepsilon)=p+\sigma \varepsilon-b+\lambda \int_{\varepsilon_{d}^{s}}^{\varepsilon_{u}} S^{s}(x) d F(x)-\beta \theta^{s} \phi\left(\theta^{s}\right) S^{s}\left(\varepsilon_{u}\right) \\
\quad-\lambda k F\left(\varepsilon_{d}^{s}\right)+\mu \check{S}^{q}(\varepsilon), \quad \varepsilon \geq \varepsilon_{d}^{s}, \\
(r+\lambda+\mu) \check{S}^{q}(\varepsilon)= \\
\quad \check{p}+\sigma \varepsilon-b+\lambda \int_{\check{\varepsilon}_{d}^{s}}^{\varepsilon_{u}} S^{s}(x) d F(x)-\beta \check{\theta}^{s} \phi\left(\check{\theta}^{s}\right) \check{S}^{q}\left(\varepsilon_{u}\right) \\
\quad-\lambda k F\left(\check{\varepsilon}_{d}^{s}\right)+\mu S^{s}(\varepsilon), \quad \varepsilon \geq \varepsilon_{d}^{s}, \\
(r+\lambda+\mu) \check{S}^{q}(\varepsilon)=\check{p}+\sigma \varepsilon-b+\lambda \int_{\check{\varepsilon}_{d}^{s}}^{\varepsilon_{u}} \check{S}^{q}(x) d F(x)-\beta \check{\theta}^{s} \phi\left(\check{\theta}^{s}\right) \check{S}^{q}\left(\varepsilon_{u}\right) \\
\quad-\lambda k F\left(\check{\varepsilon}_{d}^{s}\right)-\mu k, \quad \varepsilon_{d}>\varepsilon \geq \check{\varepsilon}_{d}^{s} .
\end{gathered}
$$

Booms and recessions have to be analyzed separately. Equation (37) may be transformed into (for details see the derivation of (29))

$$
\begin{aligned}
(r+\lambda+\mu) \check{S}^{q}(\varepsilon)= & \check{p}+\sigma \varepsilon-b-\frac{\beta c}{1-\beta} \check{\theta}^{s}+\frac{\lambda \sigma}{r+\lambda+\mu} \int_{\check{\varepsilon}_{d}^{s}}^{\varepsilon_{d}^{s}}[1-F(x)] d x \\
& +\frac{\lambda \sigma}{r+\lambda} \int_{\varepsilon_{d}^{s}}^{\varepsilon_{u}}[1-F(x)] d x-(\mu+\lambda) k, \quad \varepsilon_{d}^{s}>\varepsilon \geq \check{\varepsilon}_{d}^{s}
\end{aligned}
$$

As before, setting $\varepsilon=\check{\varepsilon}_{d}^{s}$ and using $\check{S}^{q}\left(\check{\varepsilon}_{d}^{s}\right)=-k$ gives us an implicit definition of $\check{\varepsilon}_{d}^{s}$,

$$
\begin{aligned}
\check{p}+\sigma \check{\varepsilon}_{d}^{s}=b+\frac{\beta c}{1-\beta} \check{\theta}^{s}-\frac{\lambda \sigma}{r+\lambda+\mu} \int_{\check{\varepsilon}_{d}^{s}}^{\varepsilon_{d}^{s}}[1-F(x)] d x \\
\quad-\frac{\lambda \sigma}{r+\lambda} \int_{\varepsilon_{d}^{s}}^{\varepsilon_{u}}[1-F(x)] d x-r k .
\end{aligned}
$$

Compared to the steady-state analysis, the only thing that is structurally different is the option value of keeping the job. If an idiosyncratic shock moves $\varepsilon$ into the interval $\left[\check{\varepsilon}_{d}^{s} ; \varepsilon_{d}^{s}\right)$, an additional risk arises since the job will have to be destroyed if a recessions occurs. For this reason, the value of future changes in (job-specific) productivity has to be discounted more heavily. Hence, taking into account the possibility of a decline in aggregate productivity leads to a higher threshold than in the steady-state analysis (for a given value of $\check{\theta}^{s}$ ), so that jobs will be destroyed more quickly. 
For a firm that experiences a recession, the following two equations can be obtained:

$$
\begin{aligned}
(r+\lambda+\mu) S^{s}(\varepsilon)=p+\sigma \varepsilon-b & -\frac{\beta c}{1-\beta} \theta^{s} \\
& +\frac{\lambda \sigma}{r+\lambda} \int_{\varepsilon_{d}^{s}}^{\varepsilon_{u}}[1-F(x)] d x+\mu \check{S}^{q}-(\mu+\lambda) k
\end{aligned}
$$

and

$$
p+\sigma \varepsilon_{d}^{s}=b+\frac{\beta c}{1-\beta} \theta^{s}-\frac{\lambda \sigma}{r+\lambda} \int_{\varepsilon_{d}^{s}}^{\varepsilon_{u}}[1-F(x)] d x-r k-\frac{\mu \sigma}{r+\lambda+\mu}\left(\varepsilon_{d}^{s}-\check{\varepsilon}_{d}^{s}\right) .
$$

In this case, the option value of keeping the job is larger than in the steady-state analysis (again for a given value of $\theta^{s}$ ). The last term captures the expected return if the job is not destroyed and a boom occurs.

Since $\varepsilon_{d}^{s}$ tends to be smaller and $\check{\varepsilon}_{d}^{s}$ to be larger than in the steady-state analysis, the number of jobs that is destroyed in a recession is smaller than in the case of a permanent decline from $\check{p}$ to $p .^{22}$

An rise in $\check{p}$ or $p$ reduces both threshold values for a given level of labor market tightness, see the appendix. It may be surprising that the threshold value of the 'other' regime declines as well. But suppose for example that the aggregate productivity in booms rises. For a firm that currently experiences a recession, it is profitable to tolerate lower levels of the job-specific productivity (i.e. $\varepsilon_{d}^{s}$ goes down), since this job will benefit from the higher value of $\check{p}$, too, as soon as it gets out of the recession, compare the last expression in (41). A similar argument can be found for the effect of a change in $p$ on $\check{\varepsilon}_{d}^{s}$.

It can also be shown that a rise in the firing costs $k$ lowers both threshold values.

The labor market for the low-skilled can be described analogously. One arrives at equations similar to (39) and (41) that define $\breve{\varepsilon}_{d}^{l}$ and $\varepsilon_{d}^{l}$. The two thresholds are

\footnotetext{
${ }^{22}$ This statement has to be taken cum grano salis, since one would have to differentiate the difference $\varepsilon_{d}^{s}-\breve{\varepsilon}_{d}^{s}$ with respect to $\mu$ in order to obtain a formal proof. Therefore, I will give a less rigorous reasoning. On the one hand, equations (39) and (41) show that for $\mu=0$, we have the thresholds for the two steady states characterized by $\check{p}$ and $p$. On the other hand, the two equations show that a ceteris paribus increase in $\mu$ raises $\check{\varepsilon}_{d}^{s}$ and lowers $\varepsilon_{d}^{s}$. Intuitively, an increase in $\mu$ makes the two regimes more alike, since e.g. being in a boom is less of an advantage if it is very likely to drop into a recession again soon. This suggests that $\varepsilon_{d}^{s}-\breve{\varepsilon}_{d}^{s}$ should decline with rising values of $\mu$.
} 
negative functions of $p, \check{p}$ and $k$ as well. Furthermore, it is shown in the appendix that an increase in the productivity differential $\rho$ raises both $\check{\varepsilon}_{d}^{l}$ and $\varepsilon_{d}^{l}$, i.e. the results of the steady-state analysis continue to hold.

Equation (34) characterized the tightness of the labor market for the skilled in the steady-state. In the present framework of business cycles, the same steps that led to (34) yield

$$
\phi\left(\theta^{s}\right)=\frac{c}{1-\beta} \frac{r+\lambda}{\sigma\left(\varepsilon_{u}-\varepsilon_{d}^{s}\right)-(r+\lambda) k}
$$

for the case of a recession and

$$
\phi\left(\check{\theta}^{s}\right)=\frac{c}{1-\beta} \frac{r+\lambda}{\sigma\left(\varepsilon_{u}-\check{\varepsilon}_{d}^{s}\right)-\mu \frac{\sigma\left(\varepsilon_{d}^{s}-\check{\varepsilon}_{d}^{s}\right)}{r+\lambda+\mu}-(r+\lambda) k}
$$

during booms (for details see the appendix). A comparison of the denominators reveals that the right hand side of (42) is larger than that of (43), so that $\check{\theta}^{s}>\theta^{s}$, which means that in a boom more vacancies are available relative to the number of unemployed workers. It is not possible to make a statement about the relation of the absolute numbers $\check{v}^{s}$ and $v^{s}$, since the labor market might be so tight in a boom that the condition $\check{v}^{s}<v^{s}$ holds. But the number of vacancies will increase at least immediately after a recovery sets in. This is because the unemployment rate reacts sluggishly during a recovery (due to the matching technology), whereas labor market tightness $\check{\theta}^{s}$ and $\theta^{s}$ is a variable that is path-independent and that will therefore jump directly to its higher new equilibrium level. This implies $\check{v}^{s}>v^{s}$ at least for some time after the beginning of the boom.

It seems plausible that the difference $\check{\theta}^{s}-\theta^{s}$ is a declining function of $\mu$, i.e. the more likely it is that the business cycle will turn again the smaller is the incentive to change ones employment policy. Looking at equations (42) and (43), it can be seen that for given values of $\check{\varepsilon}_{d}^{s}$ and $\varepsilon_{d}^{s}$, an increase in $\mu$ lowers $\check{\theta}^{s}$ but leaves $\theta^{s}$ unaffected. Taking into account the simultaneous reaction of the two thresholds should not lead to qualitative changes. ${ }^{23}$ The results in this paragraph also apply to the low-skill segment.

\footnotetext{
${ }^{23}$ An increase in $\mu$ is likely to increase job security during a recessions, which means a lower value of $\varepsilon_{d}^{s}$. This in turn will raise $\theta^{s}$ according to (42), which tends to close the gap between $\check{\theta}^{s}$ and $\theta^{s}$ even more.
} 


\section{Conclusions}

This paper tried to address the effects of differences in the skill level of the employed, the implications of firing costs, and the interaction of these two factors in a stochastic environment, taking into account changes both on the demand and the supply side of the labor market. To this end, the matching model of Mortensen and Pissarides (1994) has been supplemented accordingly. Apart from the productivity differential, both labor market segments were assumed to be identical in order to isolate the effects of this parameter.

Even with flexible wages, low-skill jobs were found to have on average a lower life span. In addition, unemployment and the average unemployment duration are higher among the low-skilled. Skill-biased technological change leads to a shrinkage of this sector even with flexible wages. The effect of the productivity differential on the threshold tends to be stronger if aggregate productivity is higher. A larger spread of idiosyncratic shocks reduces the negative effects of the differential.

Firing costs have a beneficial effect on job security (measured as the average life span of a job) for both skill groups, but they reduce the creation of new jobs. The effect of protecting jobs from being destroyed tends to be stronger among the skilled. Average unemployment duration increases, but the effect of higher firing costs on total employment is ambiguous, unless one assumes some form of a loss of human capital due to long-term unemployment. Finally, the analysis can be extended to include business cycles, even though some of the results from the steady-state analysis disappear or become more difficult to prove.

\section{A Appendix}

\section{A.1 Labor market for the skilled in the steady-state}




\section{A.1.1 Changes in $\beta$}

$\boldsymbol{d}^{\boldsymbol{s}}=\mathbf{0}: \quad$ Differentiation of $(11)$ with respect to $\beta$ yields

$$
\begin{aligned}
\sigma \frac{\partial \varepsilon_{d}^{s}}{\partial \beta} & =\frac{c}{(1-\beta)^{2}} \theta^{s}+\frac{\sigma \lambda}{r+\lambda}\left[1-F\left(\varepsilon_{d}^{s}\right)\right] \frac{\partial \varepsilon_{d}^{s}}{\partial \beta} \\
\Leftrightarrow \sigma \frac{\partial \varepsilon_{d}^{s}}{\partial \beta} \frac{r+\lambda F\left(\varepsilon_{d}^{s}\right)}{r+\lambda} & =c \theta^{s} \frac{1}{(1-\beta)^{2}} \\
\Rightarrow \frac{\partial \varepsilon_{d}^{s}}{\partial \beta} & >0 .
\end{aligned}
$$

$\boldsymbol{d}^{\boldsymbol{s}} \neq 0$ : Differentiation of (11) with respect to $\beta$ yields (compare (A.2))

$$
\sigma \frac{\partial \varepsilon_{d}^{s}}{\partial \beta} \frac{r+\lambda F\left(\varepsilon_{d}^{s}\right)}{r+\lambda}=\frac{c}{(1-\beta)^{2}} \theta^{s}+\frac{\beta c}{1-\beta} \frac{\partial \theta^{s}}{\partial \beta} .
$$

Differentiating (13) with respect to $\beta$ leads to

$$
\phi^{\prime}\left(\theta^{s}\right) \frac{\partial \theta^{s}}{\partial \beta}=\frac{c(r+\lambda)}{(1-\beta)^{2} \sigma\left(\varepsilon_{u}-\varepsilon_{d}^{s}\right)}+\frac{c(r+\lambda) \frac{\partial \varepsilon_{d}^{s}}{\partial \beta}}{(1-\beta) \sigma\left(\varepsilon_{u}-\varepsilon_{d}^{s}\right)^{2}} .
$$

Suppose that $\frac{\partial \theta^{s}}{\partial \beta}>0$. Since $\phi^{\prime}\left(\theta^{s}\right)<0$ we must have $\frac{\partial \varepsilon_{d}^{s}}{\partial \beta}<0$. On the other hand, (A.3) implies $\frac{\partial \varepsilon_{d}^{s}}{\partial \beta}>0$ for $\frac{\partial \theta^{s}}{\partial \beta}>0$, which is a contradiction. Hence we must have $\frac{\partial \theta^{s}}{\partial \beta}<0$.

Solving (A.4) for $\frac{\partial \theta^{s}}{\partial \beta}$, taking into account $-\eta=\phi^{\prime}\left(\theta^{s}\right) \frac{\theta^{s}}{\phi\left(\theta^{s}\right)}$, leads to

$$
\begin{aligned}
\frac{\partial \theta^{s}}{\partial \beta} & =\frac{1}{\phi^{\prime}\left(\theta^{s}\right)}\left[\frac{c(r+\lambda)}{(1-\beta)^{2} \sigma\left(\varepsilon_{u}-\varepsilon_{d}^{s}\right)}+\frac{c(r+\lambda) \frac{\partial \varepsilon_{d}^{s}}{\partial \beta}}{(1-\beta) \sigma\left(\varepsilon_{u}-\varepsilon_{d}^{s}\right)^{2}}\right] \\
& =\frac{\theta^{s}}{-\eta \phi\left(\theta^{s}\right)}\left[\frac{\phi\left(\theta^{s}\right)}{1-\beta}+\frac{\phi\left(\theta^{s}\right) \frac{\partial \varepsilon_{d}^{s}}{\partial \beta}}{\left(\varepsilon_{u}-\varepsilon_{d}^{s}\right)}\right] \\
& =\frac{-\theta^{s}}{\eta(1-\beta)}-\frac{\theta^{s} \frac{\partial \varepsilon_{d}^{s}}{\partial \beta}}{\eta\left(\varepsilon_{u}-\varepsilon_{d}^{s}\right)} .
\end{aligned}
$$

After plugging (A.5) into (A.3) one arrives at

$$
\begin{aligned}
& \sigma \frac{\partial \varepsilon_{d}^{s}}{\partial \beta} \frac{r+\lambda F\left(\varepsilon_{d}^{s}\right)}{r+\lambda}=\frac{c \theta^{s}}{(1-\beta)^{2}}-\frac{c \beta}{1-\beta}\left[\frac{\theta^{s}}{\eta(1-\beta)}+\frac{\theta^{s} \frac{\partial \varepsilon_{d}^{s}}{\partial \beta}}{\eta\left(\varepsilon_{u}-\varepsilon_{d}^{s}\right)}\right] \\
\Leftrightarrow & \frac{\partial \varepsilon_{d}^{s}}{\partial \beta} \underbrace{\left[\frac{\sigma\left(r+\lambda F\left(\varepsilon_{d}^{s}\right)\right)}{r+\lambda}+\frac{\theta^{s}}{\eta\left(\varepsilon_{u}-\varepsilon_{d}^{s}\right)}\right]}_{>0}=\frac{c \theta^{s}}{(1-\beta)^{2}}\left[1-\frac{\beta}{\eta}\right] .
\end{aligned}
$$

Therefore we have $\frac{\partial \varepsilon_{d}^{s}}{\partial \beta} \geq 0$ if $\eta \geq \beta$. 


\section{A.1.2 Changes in $c$}

$\boldsymbol{d} \boldsymbol{\theta}^{\boldsymbol{s}}=\mathbf{0}$ : Differentiation of (11) w.r.t. $c$ yields

$$
\begin{aligned}
\sigma \frac{\partial \varepsilon_{d}^{s}}{\partial c} & =\frac{\sigma \lambda}{r+\lambda}\left[1-F\left(\varepsilon_{d}^{s}\right)\right] \frac{\partial \varepsilon_{d}^{s}}{\partial c}+\frac{\beta}{1-\beta} \theta^{s} \\
\Leftrightarrow \sigma \frac{\partial \varepsilon_{d}^{s}}{\partial c} \frac{r+\lambda F\left(\varepsilon_{d}^{s}\right)}{r+\lambda} & =\frac{\beta}{1-\beta} \theta^{s} .
\end{aligned}
$$

Since the right hand side and the fraction on the left hand side are both positive, we must have $\frac{\partial \varepsilon_{d}^{s}}{\partial c}>0$.

$\boldsymbol{d} \boldsymbol{\theta}^{s} \neq 0$ : The derivatives of (11) and (13) w.r.t. $c$ are

$$
\begin{gathered}
\sigma \frac{\partial \varepsilon_{d}^{s}}{\partial c} \frac{r+\lambda F\left(\varepsilon_{d}^{s}\right)}{r+\lambda}=\frac{\beta}{1-\beta} \theta^{s}+\frac{\beta c}{1-\beta} \frac{\partial \theta^{s}}{\partial c} \\
\phi^{\prime}\left(\theta^{s}\right) \frac{\partial \theta^{s}}{\partial c}=\frac{(r+\lambda)}{\sigma(1-\beta)} \frac{\left(\varepsilon_{u}-\varepsilon_{d}^{s}\right)+c \frac{\partial \varepsilon_{d}^{s}}{\partial c}}{\left(\varepsilon_{u}-\varepsilon_{d}^{s}\right)^{2}} .
\end{gathered}
$$

Suppose that $\frac{\partial \theta^{s}}{\partial c}>0$. Again, because of $\phi^{\prime}\left(\theta^{s}\right)$ we then must have $\frac{\partial \varepsilon_{d}^{s}}{\partial c}<0$. On the other hand, the positive right hand side of (A.8) implies $\frac{\partial \varepsilon_{d}^{s}}{\partial c}>0$, which is a contradiction. Hence we must have $\frac{\partial \theta^{s}}{\partial c}<0$.

To find out the sign of $\frac{\partial \varepsilon_{d}^{s}}{\partial c}$, we plug (A.9) into (A.8) and obtain

$$
\begin{aligned}
& \sigma \frac{\partial \varepsilon_{d}^{s}}{\partial c} \frac{r+\lambda F\left(\varepsilon_{d}^{s}\right)}{r+\lambda}=\frac{\beta \theta^{s}}{1-\beta}+\frac{\beta c}{1-\beta} \frac{r+\lambda}{\sigma(1-\beta)} \frac{\left(\varepsilon_{u}-\varepsilon_{d}^{s}\right)+c \frac{\partial \varepsilon_{d}^{s}}{\partial c}}{\phi^{\prime}\left(\theta^{s}\right)\left(\varepsilon_{u}-\varepsilon_{d}^{s}\right)^{2}} \\
& \Leftrightarrow \sigma \frac{\partial \varepsilon_{d}^{s}}{\partial c}\left[\frac{r+\lambda F\left(\varepsilon_{d}^{s}\right)}{r+\lambda}-\frac{\beta c^{2}(r+\lambda)}{\phi^{\prime}\left(\theta^{s}\right) \sigma^{2}\left(\varepsilon_{u}-\varepsilon_{d}^{s}\right)^{2}(1-\beta)^{2}}\right]=\frac{\beta \theta^{s}}{1-\beta} \\
& +\frac{\beta c(r+\lambda)}{\sigma(1-\beta)^{2}\left(\varepsilon_{u}-\varepsilon_{d}^{s}\right) \phi^{\prime}\left(\theta^{s}\right)} \\
& \Leftrightarrow \sigma \frac{\partial \varepsilon_{d}^{s}}{\partial c}\left[\frac{r+\lambda F\left(\varepsilon_{d}^{s}\right)}{r+\lambda}-\frac{\beta \phi\left(\theta^{s}\right)^{2}}{\phi^{\prime}\left(\theta^{s}\right)(r+\lambda)}\right]=\frac{\beta}{1-\beta}\left[\theta^{s}+\frac{\phi\left(\theta^{s}\right)}{\phi^{\prime}\left(\theta^{s}\right)}\right] \\
& =\frac{\beta}{1-\beta} \theta^{s}\left(1-\frac{1}{\eta}\right) \\
& \Rightarrow \frac{\partial \varepsilon_{d}^{s}}{\partial c}<0
\end{aligned}
$$




\section{A.2 Labor market segment for the low-skilled}

\section{A.2.1 Derivation of (20)}

Forming the difference of (17) for $\varepsilon_{u}$ and $\varepsilon_{d}^{l}$ yields

$$
\begin{aligned}
(r+\lambda)\left[S^{l}\left(\varepsilon_{u}\right)-S^{l}\left(\varepsilon_{d}^{l}\right)\right] & =\sigma\left(\varepsilon_{u}-\varepsilon_{d}^{l}\right) \\
\Leftrightarrow \quad S^{l}\left(\varepsilon_{u}\right) & =\frac{\sigma\left(\varepsilon_{u}-\varepsilon_{d}^{l}\right)}{r+\lambda} .
\end{aligned}
$$

In addition, because of (2) and (4) we have

$$
S^{l}\left(\varepsilon_{u}\right)=\frac{1}{1-\beta} J^{l}\left(\varepsilon_{u}\right)=\frac{1}{1-\beta} \frac{c}{\phi\left(\theta^{l}\right)} .
$$

Plugging (A.12) into (A.13) and solving for $\phi\left(\theta^{l}\right)$ leads to (20).

\section{A.2.2 Changes in $\rho$}

$\boldsymbol{d} \boldsymbol{\theta}^{\boldsymbol{s}}=\mathbf{0}$ : The derivative of (18) w.r.t. $\rho$ is

$$
\begin{aligned}
\sigma\left(\frac{\partial \varepsilon_{d}^{l}}{\partial \rho}-1\right) & =\frac{\sigma \lambda}{r+\lambda}\left[1-F\left(\varepsilon_{d}^{l}\right)\right] \frac{\partial \varepsilon_{d}^{l}}{\partial \rho} \\
\Leftrightarrow \sigma \frac{\partial \varepsilon_{d}^{l}}{\partial \rho} \frac{r+\lambda F\left(\varepsilon_{d}^{l}\right)}{r+\lambda} & =\sigma \\
\Leftrightarrow \frac{\partial \varepsilon_{d}^{l}}{\partial \rho} & =\frac{r+\lambda}{r+\lambda F\left(\varepsilon_{d}^{l}\right)}>1 .
\end{aligned}
$$

From this, we obtain for the cross-derivative w.r.t. $\rho$ and $\sigma$

$$
\frac{\partial^{2} \varepsilon_{d}^{l}}{\partial \rho \partial \sigma}=\frac{-(r+\lambda) \lambda F^{\prime}\left(\varepsilon_{d}^{l}\right) \frac{\partial \varepsilon_{d}^{l}}{\partial \sigma}}{\left[r+\lambda F\left(\varepsilon_{d}^{l}\right)\right]^{2}} .
$$

It is negative for $\frac{\partial \varepsilon_{d}^{l}}{\partial \sigma}>0$. Similar steps show that $\frac{\partial^{2} \varepsilon_{d}^{l}}{\partial \rho \partial(p-b)}$ and $\frac{\partial^{2} \varepsilon_{d}^{l}}{\partial \rho \partial \beta}$.

The cross-derivative w.r.t. $\rho$ and $\lambda$ is

$$
\begin{aligned}
\frac{\partial^{2} \varepsilon_{d}^{l}}{\partial \rho \partial \lambda} & =\frac{r+\lambda F\left(\varepsilon_{d}^{l}\right)-(r+\lambda)\left[F\left(\varepsilon_{d}^{l}\right)+\lambda F^{\prime}\left(\varepsilon_{d}^{l}\right) \frac{\partial \varepsilon_{d}^{l}}{\partial \lambda}\right]}{\left[r+\lambda F\left(\varepsilon_{d}^{l}\right)\right]^{2}} \\
& =\frac{r\left[1-F\left(\varepsilon_{d}^{l}\right)\right]-(r+\lambda) \lambda F^{\prime}\left(\varepsilon_{d}^{l}\right) \frac{\partial \varepsilon_{d}^{l}}{\partial \lambda}}{\left[r+\lambda F\left(\varepsilon_{d}^{l}\right)\right]^{2}},
\end{aligned}
$$

which is positive since $F\left(\varepsilon_{d}^{l}\right)<1$ and $\frac{\partial \varepsilon_{d}^{l}}{\partial \lambda}<0$. In the same way it can be shown that the cross derivative w.r.t. $\rho$ and $r$ is negative. 
$\boldsymbol{d} \boldsymbol{\theta}^{\boldsymbol{s}} \neq \mathbf{0}$ : Differentiation of $(18)$ and $(20)$ w.r.t. $\rho$ yields

$$
\sigma \frac{\partial \varepsilon_{d}^{l}}{\partial \rho}=\frac{r+\lambda}{r+\lambda F\left(\varepsilon_{d}^{l}\right)}\left[\sigma+\frac{\beta c}{1-\beta} \frac{\partial \theta^{l}}{\partial \rho}\right] .
$$

and

$$
\phi^{\prime}\left(\theta^{l}\right) \frac{\partial \theta^{l}}{\partial \rho}=\frac{c(r+\lambda) \frac{\partial \varepsilon_{d}^{l}}{\partial \rho}}{(1-\beta) \sigma\left(\varepsilon_{u}-\varepsilon_{d}^{l}\right)^{2}},
$$

respectively. Suppose that $\frac{\partial \theta^{l}}{\partial \rho}>0$. Because of $\phi^{\prime}\left(\theta^{l}\right)<0$ we must have $\frac{\partial \varepsilon_{d}^{l}}{\partial \rho}<0$. On the other hand (A.17) and $\frac{\partial \theta^{l}}{\partial \rho}>0$ imply $\frac{\partial \varepsilon_{d}^{l}}{\partial \rho}>0$, which is a contradiction. Hence we can conclude that $\frac{\partial \theta^{l}}{\partial \rho}<0$ and - using (A.18) once more $-\frac{\partial \varepsilon_{d}^{l}}{\partial \rho}>0$ gelten.

In order to compute cross-derivatives for a variable $\theta^{l}$, we need an explicit solution for $\frac{\partial \varepsilon_{d}^{l}}{\partial \rho}$. Plugging (A.18) into (A.17) and rearranging terms leads to

$$
\begin{gathered}
\sigma \frac{\partial \varepsilon_{d}^{l}}{\partial \rho}=\frac{r+\lambda}{r+\lambda F\left(\varepsilon_{d}^{l}\right)}\left[\sigma+\frac{\beta c^{2}(r+\lambda) \frac{\partial \varepsilon_{d}^{l}}{\partial \rho}}{(1-\beta)^{2} \sigma\left(\varepsilon_{u}-\varepsilon_{d}^{l}\right)^{2} \phi^{\prime}\left(\theta^{l}\right)}\right] \\
\Leftrightarrow \quad \sigma \frac{\partial \varepsilon_{d}^{l}}{\partial \rho}\left[1-\frac{\beta}{\phi^{\prime}\left(\theta^{l}\right)\left[r+\lambda F\left(\varepsilon_{d}^{l}\right)\right]}\left[\frac{c(r+\lambda)}{(1-\beta) \sigma\left(\varepsilon_{u}-\varepsilon_{d}^{l}\right)}\right]^{2}\right]=\frac{\sigma(r+\lambda)}{r+\lambda F\left(\varepsilon_{d}^{l}\right)} \\
\Leftrightarrow \frac{\partial \varepsilon_{d}^{l}}{\partial \rho}\left(1-\frac{\beta \phi\left(\theta^{l}\right)^{2}}{\phi^{\prime}\left(\theta^{l}\right)\left[r+\lambda F\left(\varepsilon_{d}^{l}\right)\right]}\right)=\frac{r+\lambda}{r+\lambda F\left(\varepsilon_{d}^{l}\right)} \\
\frac{\partial \varepsilon_{d}^{l}}{\partial \rho}=\frac{r+\lambda}{r+\lambda F\left(\varepsilon_{d}^{l}\right)-\beta \frac{\phi\left(\theta^{l}\right)^{2}}{\phi^{\prime}\left(\theta^{l}\right)}}
\end{gathered}
$$

In the cross-derivative of $\varepsilon_{d}^{l}$ w.r.t. $\rho$ and the other parameters $\frac{\phi\left(\theta^{l}\right)^{2}}{\phi^{\prime}\left(\theta^{l}\right)}$ has to be derived w.r.t. $\theta^{l}$. Without making further assumptions, the sign of this derivative is unclear, which translates into an ambiguous sign of the cross-derivatives. Note also that contrary to the previous analysis, $\partial \varepsilon_{d}^{l} / \partial \rho$ may now be smaller than 1 because of $\phi^{\prime}\left(\theta^{l}\right)<0$. 


\section{A.2.3 Changes in $\sigma$}

$\boldsymbol{d} \boldsymbol{\theta}^{\boldsymbol{l}}=\mathbf{0}$ : $\quad$ The derivative of (18) w.r.t. $\sigma$ is

$$
\begin{aligned}
\varepsilon_{d}^{l}-\rho+\sigma \frac{\partial \varepsilon_{d}^{l}}{\partial \sigma}=- & \frac{\lambda}{r+\lambda} \int_{\varepsilon_{d}^{l}}^{\varepsilon_{u}}[1-F(x)] d x \\
& +\frac{\sigma \lambda}{r+\lambda}\left[1-F\left(\varepsilon_{d}^{l}\right)\right] \frac{\partial \varepsilon_{d}^{l}}{\partial \sigma} \\
\Leftrightarrow \sigma \frac{\partial \varepsilon_{d}^{l}}{\partial \sigma} \frac{r+\lambda F\left(\varepsilon_{d}^{l}\right)}{r+\lambda}=- & -\varepsilon_{d}^{l}+\rho-\frac{\lambda}{r+\lambda} \int_{\varepsilon_{d}^{l}}^{\varepsilon_{u}}[1-F(x)] d x \\
= & \frac{1}{\sigma}\left[p-b-\frac{\beta c}{1-\beta} \theta^{l}\right] .
\end{aligned}
$$

Hence we have $\frac{\partial \varepsilon_{d}^{l}}{\partial \sigma}>0$ if $p>b+\frac{\beta c}{1-\beta} \theta^{l}$. Qualitatively, this is the same condition as for $\frac{\partial \varepsilon_{d}^{s}}{\partial \sigma}>0$.

$\boldsymbol{d}^{\boldsymbol{l}} \neq \mathbf{0}$ : Differentiating (18) and (13) w.r.t. $\sigma$ leads to

$$
\sigma \frac{\partial \varepsilon_{d}^{l}}{\partial \sigma} \frac{r+\lambda F\left(\varepsilon_{d}^{l}\right)}{r+\lambda}=-\left(\varepsilon_{d}^{l}-\rho\right)-\frac{\lambda}{r+\lambda} \int_{\varepsilon_{d}^{l}}^{\varepsilon_{u}}[1-F(x)] d x+\frac{\beta c}{1-\beta} \frac{\partial \theta^{l}}{\partial \sigma} .
$$

and

$$
\begin{aligned}
\phi^{\prime}\left(\theta^{l}\right) \frac{\partial \theta^{l}}{\partial \sigma} & =\frac{c(r+\lambda)}{1-\beta} \frac{-\left(\varepsilon_{u}-\varepsilon_{d}^{l}-\sigma \frac{\partial \varepsilon_{d}^{l}}{\partial \sigma}\right)}{\sigma^{2}\left(\varepsilon_{u}-\varepsilon_{d}^{l}\right)^{2}} \\
& =\phi\left(\theta^{l}\right) \frac{\sigma \frac{\partial \varepsilon_{d}^{l}}{\partial \sigma}-\left(\varepsilon_{u}-\varepsilon_{d}^{l}\right)}{\sigma\left(\varepsilon_{u}-\varepsilon_{d}^{l}\right)}
\end{aligned}
$$

respectively. Making use of $-\eta=\phi^{\prime}\left(\theta^{l}\right) \frac{\left.\theta^{l}\right)}{\phi\left(\theta^{l}\right)}$ allows us to rewrite (A.24) as

$$
\begin{aligned}
\frac{\partial \theta^{l}}{\partial \sigma} \sigma\left(\varepsilon_{u}-\varepsilon_{d}^{l}\right) \frac{\eta}{\theta^{l}} & =\left(\varepsilon_{u}-\varepsilon_{d}^{l}\right)-\sigma \frac{\partial \varepsilon_{d}^{s}}{\partial \sigma} \\
\Leftrightarrow \frac{\partial \theta^{l}}{\partial \sigma} & =\frac{\theta^{l}}{\sigma \eta}-\frac{\theta^{l}}{\eta\left(\varepsilon_{u}-\varepsilon_{d}^{l}\right)} \frac{\partial \varepsilon_{d}^{l}}{\partial \sigma} .
\end{aligned}
$$

Plugging (A.23) into (A.25), one obtains

$$
\begin{aligned}
\frac{\partial \theta^{l}}{\partial \sigma}\left[\sigma\left(\varepsilon_{u}-\varepsilon_{d}^{l}\right) \frac{\eta}{\theta^{l}}+\right. & \left.\frac{r+\lambda}{r+\lambda F\left(\varepsilon_{d}^{l}\right)} \frac{\beta c}{1-\beta}\right]=\left(\varepsilon_{u}-\varepsilon_{d}^{l}\right) \\
& +\frac{r+\lambda}{r+\lambda F\left(\varepsilon_{d}^{l}\right)}\left[\varepsilon_{d}^{l}-\rho+\frac{\lambda}{r+\lambda} \int_{\varepsilon_{d}^{l}}^{\varepsilon_{u}}[1-F(x)] d x\right] .
\end{aligned}
$$


The right hand side can be simplified as follows

$$
\begin{aligned}
\frac{\partial \theta^{l}}{\partial \sigma}[\ldots] & =\varepsilon_{u}-\frac{r+\lambda}{r+\lambda F\left(\varepsilon_{d}^{l}\right)} \rho+\frac{\varepsilon_{d}^{l} \lambda\left[1-F\left(\varepsilon_{d}^{l}\right)\right]}{r+\lambda F\left(\varepsilon_{d}^{l}\right)}+\frac{\lambda}{r+\lambda F\left(\varepsilon_{d}^{l}\right)} \int_{\varepsilon_{d}^{l}}^{\varepsilon_{u}}[1-F(x) d x] \\
& =\varepsilon_{u}-\frac{r+\lambda}{r+\lambda F\left(\varepsilon_{d}^{l}\right)} \rho+\frac{\lambda\left[1-F\left(\varepsilon_{d}^{l}\right)\right]}{r+\lambda F\left(\varepsilon_{d}^{l}\right)}\left[\varepsilon_{d}^{l}+\int_{\varepsilon_{d}^{l}}^{\varepsilon_{u}} \frac{1-F(x)}{1-F\left(\varepsilon_{d}^{l}\right)} d x\right] \\
& =\varepsilon_{u}-\frac{r+\lambda}{r+\lambda F\left(\varepsilon_{d}^{l}\right)} \rho+\frac{\lambda\left[1-F\left(\varepsilon_{d}^{l}\right)\right]}{r+\lambda F\left(\varepsilon_{d}^{l}\right)} E\left(\varepsilon \mid \varepsilon \geq \varepsilon_{d}^{l}\right) .
\end{aligned}
$$

Since the term in brackets on the left hand side is positive, the right hand side determines the sign of $\frac{\partial \theta^{l}}{\partial \sigma}$. It is positive, as long as $\rho$ is not too large (note that $\varepsilon_{u}>E\left(\varepsilon \mid \varepsilon \geq \varepsilon_{d}^{l}\right)>0$, since $\left.E(\varepsilon)=0\right)$. A sufficient condition for a positive derivative is e.g. $E\left(\varepsilon \mid \varepsilon \geq \varepsilon_{d}^{l}\right)>\rho$, for (A.28) may be rewritten as

$$
\frac{\partial \theta^{s}}{\partial \sigma}[\ldots]=\varepsilon_{u}-\rho+\frac{\lambda\left[1-F\left(\varepsilon_{d}^{l}\right)\right]}{r+\lambda F\left(\varepsilon_{d}^{l}\right)}\left[E\left(\varepsilon \mid \varepsilon \geq \varepsilon_{d}^{l}\right)-\rho\right] .
$$

The sign of $\frac{\partial \varepsilon_{d}^{l}}{\partial \sigma}$ can be determined by plugging (A.26) into (A.23). One obtains

$$
\begin{aligned}
& \sigma \frac{\partial \varepsilon_{d}^{l}}{\partial \sigma} \frac{r+\lambda F\left(\varepsilon_{d}^{l}\right)}{r+\lambda}=-\left(\varepsilon_{d}^{l}-\rho\right)-\frac{\lambda}{r+\lambda} \int_{\varepsilon_{d}^{l}}^{\varepsilon_{u}}[1-F(x)] d x \\
& +\frac{\beta c}{1-\beta}\left[\frac{\theta^{l}}{\sigma \eta}-\frac{\theta^{l} \frac{\partial \varepsilon_{d}^{l}}{\partial \sigma}}{\eta\left(\varepsilon_{u}-\varepsilon_{d}^{l}\right)}\right] \\
& \Leftrightarrow \frac{\partial \varepsilon_{d}^{l}}{\partial \sigma}\left[\frac{\sigma\left(r+\lambda F\left(\varepsilon_{d}^{l}\right)\right)}{r+\lambda}+\frac{\beta c \theta^{l}}{(1-\beta) \eta\left(\varepsilon_{u}-\varepsilon_{d}^{l}\right)}\right]= \\
& \frac{1}{\sigma}\left[-\sigma\left(\varepsilon_{d}^{l}-\rho\right)-\frac{\sigma \lambda}{r+\lambda} \int_{\varepsilon_{d}^{l}}^{\varepsilon_{u}}[1-F(x)] d x+\frac{\beta c}{1-\beta} \theta^{l}+\frac{\beta c}{1-\beta} \theta^{s}\left(\frac{1}{\eta}-1\right)\right] \\
& \left.=\frac{1}{\sigma}\left[p-b+\frac{\beta c}{1-\beta} \theta^{l}\left(\frac{1}{\eta}-1\right)\right)\right] .
\end{aligned}
$$

The term in brackets on the left hand side is positive, so that $p \geq b$ is a sufficient condition for a positive sign of $\frac{\partial \varepsilon_{d}^{l}}{\partial \sigma}$.

\section{A.3 Introducing firing costs}

Derivation of equation (29): Differentiating (28) w.r.t. $\varepsilon$ yields

$$
S^{q \prime}(\varepsilon)=\frac{\sigma}{r+\lambda} .
$$


In (28) the term in brackets can be transformed as follows, using integration by parts:

$$
\begin{aligned}
\int_{\varepsilon_{d}^{s}}^{\varepsilon_{u}} S^{s}(x) d F(x)-k F\left(\varepsilon_{d}^{s}\right)= & {\left[S^{s}(x) F(x)\right]_{\varepsilon_{d}^{s}}^{\varepsilon_{u}}-\int_{\varepsilon_{d}^{s}}^{\varepsilon_{u}} S^{q \prime}(x) F(x) d x-k F\left(\varepsilon_{d}^{s}\right) } \\
= & S^{s}\left(\varepsilon_{u}\right) \cdot 1-(-k) F\left(\varepsilon_{d}^{s}\right) \\
& \quad-\frac{\sigma}{r+\lambda} \int_{\varepsilon_{d}^{s}}^{\varepsilon_{u}} F(x) d x-k F\left(\varepsilon_{d}^{s}\right) \\
= & \int_{\varepsilon_{d}^{s}}^{\varepsilon_{u}} S^{q \prime}(x) d x-k-\frac{\sigma}{r+\lambda} \int_{\varepsilon_{d}^{s}}^{\varepsilon_{u}} F(x) d x \\
= & \frac{\sigma}{r+\lambda} \int_{\varepsilon_{d}^{s}}^{\varepsilon_{u}}[1-F(x)] d x-k
\end{aligned}
$$

One obtains the last but one line by noting that $S^{s}(\varepsilon)$ takes on the value $-k$ for $\varepsilon \leq \varepsilon_{d}^{s}$, in other words $-k$ is the constant of integration.

In addition, (2), (23) and $S^{s}\left(\varepsilon_{u}\right)>0$ imply

$$
S^{s}\left(\varepsilon_{u}\right)=\frac{c}{(1-\beta) \phi\left(\theta^{s}\right)} .
$$

Plugging (A.32) and (A.33) into (28) leads to (29).

\section{Changes in $k$ :}

$d \theta^{s}=0: \quad$ Differentiation of $(30)$ w.r.t. $k$ yields

$$
\begin{aligned}
\sigma \frac{\partial \varepsilon_{d}^{s}}{\partial k} & =\frac{\sigma}{r+\lambda}\left[1-F\left(\varepsilon_{d}^{s}\right)\right] \frac{\partial \varepsilon_{d}^{s}}{\partial k}-r \\
\Leftrightarrow \quad \sigma \frac{\partial \varepsilon_{d}^{s}}{\partial k} & =-\frac{r+\lambda}{r+\lambda F\left(\varepsilon_{d}^{s}\right)} r<0 .
\end{aligned}
$$

$d \theta^{s} \neq 0$ : The derivatives of $(30)$ and (34) w.r.t. $k$ are

$$
\sigma \frac{\partial \varepsilon_{d}^{s}}{\partial k}=\frac{r+\lambda}{r+\lambda F\left(\varepsilon_{d}^{s}\right)}\left(-r+\frac{\beta c}{1-\beta} \frac{\partial \theta^{s}}{\partial k}\right) .
$$

and

$$
\phi^{\prime}\left(\theta^{s}\right) \frac{\partial \theta^{s}}{\partial k}=\frac{c(r+\lambda)}{1-\beta} \frac{\sigma \frac{\partial \varepsilon_{d}^{s}}{\partial k}+(r+\lambda)}{\left[\sigma\left(\varepsilon_{u}-\varepsilon_{d}^{s}\right)-(r+\lambda) k\right]^{2}}
$$


Suppose that $\frac{\partial \theta^{s}}{\partial k}>0$. Because of $\phi^{\prime}(\cdot)<0$ we must have $\frac{\partial \varepsilon_{d}^{s}}{\partial k}<0$ for the right hand side of (A.36) to be negative as well. If we have instead $\frac{\partial \theta^{s}}{\partial k}<0$, it follows from (A.35) that we must have $\frac{\partial \varepsilon_{d}^{s}}{\partial k}<0$ again. In order to determine the sign of $\frac{\partial \theta^{s}}{\partial k}$, we plug (A.35) into (A.36) and obtain

$$
\begin{aligned}
\frac{\partial \theta^{s}}{\partial k}\left[\phi^{\prime}\left(\theta^{s}\right)-\right. & \frac{\beta c^{2}(r+\lambda)^{2}}{\left.(1-\beta)^{2}\left[r+\lambda F\left(\varepsilon_{d}^{s}\right)\right]\left[\sigma\left(\varepsilon_{u}-\varepsilon_{d}^{s}\right)-(r+\lambda) k\right]^{2}\right]}= \\
& \frac{c(r+\lambda)^{2}}{(1-\beta)\left[\sigma\left(\varepsilon_{u}-\varepsilon_{d}^{s}\right)-(r+\lambda) k\right]^{2}}\left[-\frac{r}{r+\lambda F\left(\varepsilon_{d}^{s}\right)} r+1\right] .
\end{aligned}
$$

The term in brackets on the left hand side of (A.37) is negative, whereas the right hand side is positive, so that we must have $\frac{\partial \theta^{s}}{\partial k}<0$.

The cross-derivative $\boldsymbol{\partial}\left(\frac{\partial \boldsymbol{\varepsilon}_{\boldsymbol{d}}^{l}}{\boldsymbol{\partial} \boldsymbol{k}}\right) / \boldsymbol{\partial} \boldsymbol{\rho}$ : Solving equations (A.35) and (A.36) for $\partial \varepsilon_{d}^{l} / \partial \rho$ yields (A.20), which may be differentiated w.r.t. $k$ :

$$
\frac{\partial^{2} \varepsilon_{d}^{l}}{\partial \rho \partial k}=\frac{-(r+\lambda)\left[\lambda F^{\prime}\left(\varepsilon_{d}^{l}\right) \frac{\partial \varepsilon_{d}^{l}}{\partial k}-\beta d\left(\frac{\phi\left(\theta^{l}\right)^{2}}{\phi^{\prime}\left(\theta^{l}\right)}\right) / d \theta^{l} \frac{\partial \theta^{l}}{\partial k}\right]}{r+\lambda F\left(\varepsilon_{d}^{l}\right)-\beta \frac{\phi\left(\theta^{l}\right)^{2}}{\phi^{\prime}\left(\theta^{l}\right)}}
$$

Note that because $\frac{\partial \varepsilon_{d}^{l}}{\partial k}, \frac{\partial \theta^{l}}{\partial k}<0$, the cross-derivative will be positive if

$$
\begin{aligned}
\frac{d\left(\frac{\phi\left(\theta^{l}\right)^{2}}{\phi^{\prime}\left(\theta^{l}\right)}\right)}{d \theta^{l}} & =\frac{2 \phi\left(\theta^{l}\right) \phi^{\prime}\left(\theta^{l}\right)^{2}-\phi\left(\theta^{l}\right)^{2} \phi^{\prime \prime}\left(\theta^{l}\right)}{\phi^{\prime}\left(\theta^{l}\right)^{2}} \\
& =\phi\left(\theta^{l}\right) \frac{2 \phi^{\prime}\left(\theta^{l}\right)^{2}-\phi\left(\theta^{l}\right) \phi^{\prime \prime}\left(\theta^{l}\right)}{\phi^{\prime}\left(\theta^{l}\right)^{2}}
\end{aligned}
$$

is negative. Assume a Cobb-Douglas matching function $m\left(v^{l}, u^{l}\right)=v^{\alpha} u^{1-\alpha}$. Then we have $\phi\left(\theta^{l}\right)=\theta^{\alpha-1}, \phi^{\prime}\left(\theta^{l}\right)=(\alpha-1) \theta^{\alpha-2}<0$ and $\phi^{\prime \prime}\left(\theta^{l}\right)=(\alpha-1)(\alpha-$ 2) $\theta^{\alpha-3}>0$. Plugging this into the numerator yields $-\alpha \theta^{2 \alpha-4}<0$, so that (A.39) is negative, as needed.

\section{A.4 Business cycle fluctuations}

\section{A.4.1 Changes in aggregate productivity $(\check{p}$ and $p)$}

A change in $\check{p}$ (or $p$ ) has effects on $\check{\varepsilon}_{d}^{s}$ as well as $\varepsilon_{d}^{s}$. Therefore, both (39) and (41) have to be differentiated w.r.t. $\check{p}$ (or $p$ ). Since the calculations for $\check{p}$ and $p$ are 
very similar, only the steps for $\check{p}$ will be presented. Differentiation of (39) and (41) w.r.t. $\check{p}$ yields

$$
\begin{aligned}
\sigma \frac{\partial \check{\varepsilon}_{d}^{s}}{\partial \check{p}}=-1-\frac{\lambda \sigma}{r+\mu+\lambda}\left\{\left[1-F\left(\varepsilon_{d}^{s}\right)\right] \frac{\partial \varepsilon_{d}^{s}}{\partial \check{p}}-\left[1-F\left(\check{\varepsilon}_{d}^{s}\right)\right] \frac{\partial \check{\varepsilon}_{d}^{s}}{\partial \check{p}}\right\} \\
+\frac{\lambda \sigma}{r+\lambda}\left[1-F\left(\varepsilon_{d}^{s}\right)\right] \frac{\partial \varepsilon_{d}^{s}}{\partial \check{p}} \\
\Leftrightarrow \sigma \frac{\partial \check{\varepsilon}_{d}^{s}}{\partial \check{p}} \frac{r+\mu+\lambda F\left(\check{\varepsilon}_{d}^{s}\right)}{r+\mu+\lambda}=-1+\frac{\lambda \mu}{(r+\lambda)(r+\mu+\lambda)}\left[1-F\left(\varepsilon_{d}^{s}\right)\right] \frac{\partial \varepsilon_{d}^{s}}{\partial \check{p}} .
\end{aligned}
$$

and

$$
\begin{gathered}
\sigma \frac{\partial \varepsilon_{d}^{s}}{\partial \check{p}}=-\frac{\lambda \sigma}{r+\lambda}\left[1-F\left(\varepsilon_{d}^{s}\right)\right] \frac{\partial \varepsilon_{d}^{s}}{\partial \check{p}}-\frac{\mu \sigma}{r+\lambda+\mu}\left[\frac{\partial \varepsilon_{d}^{s}}{\partial \check{p}}-\frac{\partial \check{\varepsilon}_{d}^{s}}{\partial \check{p}}\right] \\
\Leftrightarrow \sigma \frac{\partial \varepsilon_{d}^{s}}{\partial \check{p}}\left[\frac{r+\lambda F\left(\varepsilon_{d}^{s}\right)}{r+\lambda}+\frac{\mu}{r+\lambda+\mu}\right]=\frac{\mu \sigma}{r+\lambda+\mu} \frac{\partial \check{\varepsilon}_{d}^{s}}{\partial \check{p}} .
\end{gathered}
$$

Since the expression in brackets on the left hand side of (A.41) is positive, the two derivatives $\frac{\partial \varepsilon_{d}^{s}}{\partial \ddot{p}}$ and $\frac{\partial \check{\varepsilon}_{d}^{s}}{\partial p}$ have the same sign. Plugging (A.41) into (A.40) leads to

$$
\begin{aligned}
& \sigma \frac{\partial \check{\varepsilon}_{d}^{s}}{\partial \check{p}} \frac{r+\mu+\lambda F\left(\check{\varepsilon}_{d}^{s}\right)}{r+\mu+\lambda}= \\
& \quad-1+\frac{\mu^{2} \lambda \sigma\left[1-F\left(\varepsilon_{d}^{s}\right)\right]}{(r+\mu+\lambda)\left[\left(r+\lambda F\left(\varepsilon_{d}^{s}\right)\right)(r+\mu+\lambda)+\mu(r+\lambda)\right]} \frac{\partial \check{\varepsilon}_{d}^{s}}{\partial \check{p}} \\
& \quad \Leftrightarrow \sigma \frac{\partial \check{\varepsilon}_{d}^{s}}{\partial \check{p}} \frac{\Psi}{(r+\mu+\lambda)\left[\left(r+\lambda F\left(\varepsilon_{d}^{s}\right)\right)(r+\mu+\lambda)+\mu(r+\lambda)\right]}=-1
\end{aligned}
$$

with

$$
\begin{aligned}
\Psi= & {\left[r+\mu+\lambda F\left(\check{\varepsilon}_{d}^{s}\right)\right]\left\{\left[r+\lambda F\left(\varepsilon_{d}^{s}\right)\right](r+\mu+\lambda)+\mu(r+\lambda)\right\}-\mu^{2} \lambda\left[1-F\left(\varepsilon_{d}^{s}\right)\right] } \\
=[r & \left.+\lambda F\left(\check{\varepsilon}_{d}^{s}\right)\right]\left\{\left[r+\lambda F\left(\varepsilon_{d}^{s}\right)\right](r+\lambda+\mu)+\mu(r+\lambda)\right\} \\
& +\mu\left[r+\lambda F\left(\varepsilon_{d}^{s}\right)\right](r+\mu+\lambda)+\mu^{2}\left(r+\lambda F\left(\varepsilon_{d}^{s}\right)\right)>0,
\end{aligned}
$$

so that we have $\frac{\partial \check{\varepsilon}_{d}^{s}}{\partial \ddot{p}}, \frac{\partial \varepsilon_{d}^{s}}{\partial \ddot{p}}<0$. 


\section{A.4.2 Changes in the productivity-differential $\rho$}

An increase in $\boldsymbol{\rho}$ for $\boldsymbol{d} \check{\boldsymbol{\theta}}^{l}=\boldsymbol{d} \boldsymbol{\theta}^{l}=\mathbf{0}$ : Differentiation w.r.t. $\rho$ of the equations for the low-skilled that correspond to (39) and (41) yields

$$
\begin{gathered}
\sigma \frac{\partial \check{\varepsilon}_{d}^{l}}{\partial \rho}=\sigma-\frac{\lambda \sigma}{r+\mu+\lambda}\left\{\left[1-F\left(\varepsilon_{d}^{l}\right)\right] \frac{\partial \varepsilon_{d}^{l}}{\partial \rho}-\left[1-F\left(\check{\varepsilon}_{d}^{l}\right)\right] \frac{\partial \check{\varepsilon}_{d}^{l}}{\partial \rho}\right\} \\
+\frac{\lambda \sigma}{r+\lambda}\left[1-F\left(\varepsilon_{d}^{l}\right)\right] \frac{\partial \varepsilon_{d}^{l}}{\partial \rho} \\
\Leftrightarrow \quad \sigma \frac{\partial \check{\varepsilon}_{d}^{l}}{\partial \rho} \frac{r+\mu+\lambda F\left(\check{\varepsilon}_{d}^{l}\right)}{r+\mu+\lambda}=\sigma+\frac{\lambda \mu \sigma}{(r+\lambda)(r+\mu+\lambda)}\left[1-F\left(\varepsilon_{d}^{l}\right)\right] \frac{\partial \varepsilon_{d}^{l}}{\partial \rho} .
\end{gathered}
$$

and

$$
\begin{gathered}
\sigma \frac{\partial \varepsilon_{d}^{l}}{\partial \rho}=\sigma+\frac{\lambda \sigma}{r+\lambda}\left[1-F\left(\varepsilon_{d}^{l}\right)\right] \frac{\partial \varepsilon_{d}^{l}}{\partial \rho}-\frac{\mu \sigma}{r+\mu+\lambda}\left[\frac{\partial \varepsilon_{d}^{l}}{\partial \rho}-\frac{\partial \check{\varepsilon}_{d}^{l}}{\partial \rho}\right] \\
\Leftrightarrow \sigma \frac{\partial \varepsilon_{d}^{l}}{\partial \rho} \frac{\Upsilon}{(r+\mu+\lambda)(r+\lambda)}=\sigma+\frac{\mu \sigma}{r+\mu+\lambda} \frac{\partial \check{\varepsilon}_{d}^{l}}{\partial \rho}
\end{gathered}
$$

with

$$
\Upsilon=\left[r+\lambda F\left(\varepsilon_{d}^{l}\right)\right](r+\mu+\lambda)+\mu(r+\lambda)
$$

Suppose that $\partial \check{\varepsilon}_{d}^{l} / \partial \rho<0$. Because of (A.45), $\partial \varepsilon_{d}^{l} / \partial \rho$ then has to be negative as well. If, instead, $\partial \check{\varepsilon}_{d}^{l} / \partial \rho$ is positive, equation (A.46) shows that $\partial \varepsilon_{d}^{l} / \partial \rho$ must be positive, too. The same is true if one takes the sign of $\partial \varepsilon_{d}^{l} / \partial \rho$ as a starting point. This leads to the conclusion that the two derivatives have the same sign.

In order to determine the sign of $\partial \breve{\varepsilon}_{d}^{l} / \partial \rho$, we plug (A.46) into (A.45) and obtain

$$
\begin{aligned}
& \sigma \frac{\partial \check{\varepsilon}_{d}^{l}}{\partial \rho} \frac{r+\mu+\lambda F\left(\check{\varepsilon}_{d}^{l}\right)}{r+\mu+\lambda}=\sigma+\frac{\lambda \mu \sigma\left[1-F\left(\varepsilon_{d}^{l}\right)\right]}{\Upsilon}\left[1+\frac{\mu}{r+\mu+\lambda} \frac{\partial \check{\varepsilon}_{d}^{l}}{\partial \rho}\right] \\
& \Leftrightarrow \quad \sigma \frac{\partial \check{\varepsilon}_{d}^{l}}{\partial \rho}\left[\frac{r+\mu+\lambda F\left(\check{\varepsilon}_{d}^{l}\right)}{r+\mu+\lambda}-\frac{\lambda \mu^{2}\left[1-F\left(\varepsilon_{d}^{l}\right)\right]}{\Upsilon(r+\mu+\lambda)}\right]=\sigma\left[1+\frac{\mu \lambda\left[1-F\left(\varepsilon_{d}^{l}\right)\right]}{\Upsilon}\right] .
\end{aligned}
$$

Similar steps as in the context of equation (A.44) show that the expression in brackets on the left hand side is positive. Since the right hand side is postive as well, we can conclude that $\partial \check{\varepsilon}_{d}^{l} / \partial \rho, \partial \varepsilon_{d}^{l} / \partial \rho>0$. 


\section{A.4.3 Derivation of equations (42) and (43)}

Equation (42): Subtracting (36) for $\varepsilon=\varepsilon_{d}^{s}$ from the same equation for a $\varepsilon \geq \varepsilon_{d}^{s}$ yields

$$
(r+\mu+\lambda)\left[S^{s}(\varepsilon)+k\right]=\sigma\left(\varepsilon-\varepsilon_{d}^{s}\right)+\mu\left[\check{S}^{q}(\varepsilon)-\check{S}^{q}\left(\varepsilon_{d}^{s}\right)\right] .
$$

Doing the same with equation (36) leads to

$$
(r+\mu+\lambda)\left[\check{S}^{q}(\varepsilon)-\check{S}^{q}\left(\varepsilon_{d}^{s}\right)\right]=\sigma\left(\varepsilon-\varepsilon_{d}^{s}\right)+\mu\left[S^{s}(\varepsilon)+k\right] .
$$

After plugging (A.50) into (A.49), one arrives at

$$
\begin{gathered}
(r+\mu+\lambda)\left[S^{s}(\varepsilon)+k\right]=\sigma\left(\varepsilon-\varepsilon_{d}^{s}\right)+\frac{\mu}{r+\mu+\lambda}\left\{\sigma\left(\varepsilon-\varepsilon_{d}^{s}\right)+\mu\left[S^{s}(\varepsilon)+k\right]\right\} \\
\Leftrightarrow\left[S^{s}(\varepsilon)+k\right] \frac{(r+\mu+\lambda)^{2}-\mu^{2}}{r+\mu+\lambda}=\sigma\left(\varepsilon-\varepsilon_{d}^{s}\right) \frac{r+2 \mu+\lambda}{r+\mu+\lambda} \\
\Leftrightarrow S^{s}(\varepsilon)=\frac{\sigma\left(\varepsilon-\varepsilon_{d}^{s}\right)}{r+\lambda}-k
\end{gathered}
$$

Setting $\varepsilon$ equal to $\varepsilon_{u}$ and making use of (23)and (2) yields equation (42):

$$
\begin{aligned}
S^{s}\left(\varepsilon_{u}\right) & =\frac{c}{(1-\beta) \phi\left(\theta^{s}\right)}=\frac{\sigma\left(\varepsilon_{u}-\varepsilon_{d}^{l}\right)}{r+\lambda}-k \\
\Leftrightarrow \phi\left(\theta^{s}\right) & =\frac{c(r+\lambda)}{1-\beta} \frac{1}{\sigma\left(\varepsilon-\varepsilon_{d}^{l}\right)-(r+\lambda) k} .
\end{aligned}
$$

Equation (43): Subtracting (37) for $\varepsilon=\check{\varepsilon}_{d}^{s}$ from (36) for a $\varepsilon \geq \varepsilon_{d}^{s}$ yields

$$
\begin{aligned}
& (r+\mu+\lambda)\left[\check{S}^{q}(\varepsilon)+k\right]=\sigma\left(\varepsilon-\check{\varepsilon}_{d}^{s}\right)+\mu\left[S^{s}(\varepsilon)+k\right] \\
& \Leftrightarrow \check{S}^{q}(\varepsilon)=\frac{\sigma\left(\varepsilon-\check{\varepsilon}_{d}^{s}\right)}{r+\mu+\lambda}+\frac{\mu}{r+\mu+\lambda} \frac{\sigma\left(\varepsilon-\varepsilon_{d}^{s}\right)}{r+\lambda}-k .
\end{aligned}
$$

Again, setting $\varepsilon$ equal to $\varepsilon_{u}$ and making use of (23), (2) and (A.51) we arrive at equation (43):

$$
\begin{gathered}
\check{S}^{q}\left(\varepsilon_{u}\right)=\frac{c}{(1-\beta) \phi\left(\check{\theta}^{s}\right)}=\frac{\sigma\left(\varepsilon_{u}-\check{\varepsilon}_{d}^{s}\right)}{r+\mu+\lambda}+\frac{\mu}{r+\mu+\lambda} \frac{\sigma\left(\varepsilon_{u}-\varepsilon_{d}^{s}\right)}{r+\lambda}-k \\
\Leftrightarrow \phi\left(\check{\theta}^{s}\right)=\frac{c(r+\lambda)}{1-\beta} \frac{1}{\frac{\sigma\left(\varepsilon_{u}-\check{\varepsilon}_{d}^{s}\right)(r+\lambda)}{r+\mu \lambda}+\frac{\mu \sigma\left(\varepsilon_{u}-\varepsilon_{d}^{s}\right)}{r+\lambda+\mu}-(r+\lambda) k} \\
\Leftrightarrow \phi\left(\check{\theta}^{s}\right)=\frac{c(r+\lambda)}{1-\beta} \frac{1}{\sigma\left(\varepsilon_{u}-\check{\varepsilon}_{d}^{s}\right)-\mu \frac{\sigma\left(\varepsilon_{d}^{s}-\check{\varepsilon}_{d}^{s}\right)}{r+\lambda+\mu}-(r+\lambda) k} .
\end{gathered}
$$




\section{References}

Bentolila, Samuel and Gilles Sain-Paul, "The Macroeconomic Impact of Flexible Labor Contracts, with an Application to Spain," European Economic Review, 1992, 36 (5), 1013-1053.

and Giuseppe Bertola, "Firing Costs and Labour Demand: How Bad is Eurosclerosis?," Review of Economic Studies, 1990, 57, 381-402.

Burda, Michael and Charles Wyplosz, "Gross Worker and Job Flows in Europe," European Economic Review, 1994, 38 (6), 1287 - 1315.

Coe, David T. and Dennis J. Snower, "Policy Complementarities: The Case for Fundamental Labor Market Reform," IMF Staff Papers, 1997, 44 (1), 135.

Garibaldi, Pietro, "The Asymetric Effects of Monetary Policy on Job Creation and Destruction," IMF Staff Papers, 1997, 44 (4), 557-584.

Hamermesh, Daniel S. and Gerard A. Pfann, "Adjustment Costs in Factor Demand," Journal of Economic Literature, 1996, XXXIV (3), 1264-1292.

Huizinga, Frederik and Fabio Schiantarelli, "Dynamics and Asymmetric Adjustment in Insider-Outsider Models," Economic Journal, 1992, 102 (415), 1451-1466.

Mortensen, Dale T. and Christopher A. Pissarides, "Job Creation and Job Destruction in the Theory of Unemployment," Review of Economic Studies, 1994, 61 (3), 397-415.

Pissarides, Christopher A., "Loss of Skill During Unemployment and the Persistence of Employment Shocks," Quarterly Journal of Economics, 1992, 107 (4), 1371-1391.

Ross, Matthias, Unsicherheit und Arbeitsnachfrage - Eine Theoretische und Empirische Analyse Des Warenproduzierenden Gewerbes in Deutschland, Frankfurt am Main, Berlin, Bern New York, Paris, Wien: Peter Lang, 1998.

Saint-Paul, Gilles, "Are the Unemployed Unemployable?," European Economic Review, 1996, 40 (7), 1501-1519. 
__ Dual Labor Markets - A Macroeconomic Perspective, Cambridge, MA: The MIT Press, 1996. 